\title{
Mediterranean diet and its components in relation to all-cause mortality: meta-analysis
}

\author{
Dimitra Eleftheriou ${ }^{1,2}$, Vassiliki Benetou ${ }^{1}$, Antonia Trichopoulou ${ }^{1,3}$, Carlo La Vecchia ${ }^{4}$ and Christina Bamia ${ }^{1 *}$ \\ ${ }^{1}$ Department of Hygiene, Epidemiology and Medical Statistics, Medical School, National and Kapodistrian University of \\ Athens, 75 Mikras Asias Street, Athens 115 27, Greece \\ ${ }^{2}$ School of Mathematics and Statistics, University of Glasgow, University Place, Glasgow G12 8QQ, UK \\ ${ }^{3}$ Hellenic Health Foundation, Kaisareias 13 and Alexandroupoleos Street, Athens 115 27, Greece \\ ${ }^{4}$ Department of Clinical Sciences and Community Health, University of Milan, Via Vanzetti 5, 20133 Milan, Italy
}

(Submitted 18 April 2018 - Final revision received 19 July 2018 - Accepted 3 August 2018)

\section{Abstract}

The beneficial association of the Mediterranean diet (MedDiet) with longevity has been consistently demonstrated, but the associations of MedDiet components have not been accordingly evaluated. We performed an updated meta-analysis of prospective cohort studies published up to 31 December 2017, to quantify the association of adherence to MedDiet, expressed as an index/score (MDS) and of its components with all-cause mortality. We estimated summary relative risks (SRR) and $95 \% \mathrm{CI}$ using random effects models. On the basis of thirty studies ( 225600 deaths), SRR for the study-specific highest/lowest and per 1sD MDS increment were 0.79 (95\% CI $0 \cdot 77,0 \cdot 81, I^{2}=42 \%, P$-heterogeneity $0 \cdot 02$ ) and $0.92\left(95 \%\right.$ CI $0.90,0.94, I^{2} 56 \%, P$-heterogeneity $\left.<0.01\right)$, respectively. Inversely, statistically significant associations were evident in stratified analyses by country, MDS range and publication year, with some evidence for heterogeneity across countries overall ( $P$ heterogeneity 0.011), as well as across European countries $(P=0 \cdot 018)$. Regarding MDS components, relatively stronger and statistically significant inverse associations were highlighted for moderate/none-excessive alcohol consumption (0.86, 95\% CI 0.77, 0.97) and for above/ below-the-median consumptions of fruit $(0 \cdot 88,95 \% \mathrm{CI} 0 \cdot 83,0 \cdot 94)$ and vegetables $(0 \cdot 94,95 \% \mathrm{CI} 0 \cdot 89,0 \cdot 98)$, whereas a positive association was apparent for above/below-the-median intake of meat (1.07, $95 \%$ CI 1.01, 1.13). Our meta-analyses confirm the inverse association of MedDiet with mortality and highlight the dietary components that influence mostly this association. Our results are important for better understanding the role of MedDiet in health and proposing dietary changes to effectively increase adherence to this healthy dietary pattern.

Key words: Mediterranean diet: Mortality: Meta-analyses: Reviews

The association of the Mediterranean diet (MedDiet) with health and longevity has been consistently demonstrated in a large number of observational studies, as well as in some randomised clinical trials $(\mathrm{RCT})^{(1-3)}$. Comprehensive meta-analyses have reported a substantial decrease in the incidence of, and mortality from, various chronic diseases associated with higher adherence to the MedDiet ${ }^{(4,5)}$. Recently, an umbrella review classified the accumulated evidence as 'robust' regarding the protective role of the MedDiet in reducing the risk of total mortality, CVD overall, CHD, myocardial infarction, cancer, neurodegenerative diseases and diabetes mellitus ${ }^{(6)}$.

Despite a general consensus on the beneficial influence of this diet on health, several issues remain unsolved. First, quantification of this diet has been carried out through indices and scores, which are different from each other with respect to the constructing algorithms, range of values, as well as food groups/nutrients that are included as components in these indices. Second, the relative role of the individual components of the MedDiet in the association with health outcomes of the MedDiet in toto has not been fully evaluated, except for a small number of studies ${ }^{(5,7,8)}$. Third, in the vast majority of the studies, the cut-offs that are used to define high $v$. low adherence to the components of the MedDiet are population-specific; that is, they vary across populations according to the amount of consumption of each food group/nutrient composing the score. Recently, Sofi et $a l .{ }^{(4)}$ attempted to define common cut-offs by constructing a literature-based MedDiet score, which has been tested so far in a few studies ${ }^{(9,10)}$.

The most recent meta-analysis on the association of the MedDiet with all-cause mortality ${ }^{(4)}$ included investigations published up to June 2013. We have therefore performed an updated meta-analysis of prospective cohort studies aiming to (1) quantify the association of the MedDiet with all-cause mortality among adults using all available data up to January 2018, (2) to explore

Abbreviations: HR, hazard ratio; MedDiet, Mediterranean diet; MDS, Mediterranean diet score; RR, relative risk.

* Corresponding author: Associate Professor C. Bamia, email cbamia@med.uoa.gr 
the heterogeneity across studies and (3) to evaluate the relative contribution of the individual components of this diet in the overall association of the MedDiet with mortality.

\section{Methods}

\section{Search strategy}

As a starting set, we used those studies published up to June 2013, which were included in the latest meta-analysis by Sofi et $a l^{(4)}$. Furthermore, in December 2017, we carried out a literature search in the PubMed database using the following keywords: (Mediterranean diet [Title/Abstract]) AND ((mortality [Title/Abstract]) OR (survival [Title/Abstract]) OR (longevity[Title/ Abstract]) OR (death[Title/Abstract])) AND ('2013/07/01'[Date Publication]:'2017/12/31'[Date - Publication]). Studies were included if they (i) had a cohort design or were case-control studies nested within cohorts, (ii) used a MedDiet index/score (labelled as MDS hereafter) defined a priori, (iii) investigated allcause mortality and examined associations with MDS and (iv) provided the RR estimate (i.e. risk or rate ratios (RRa) hazard ratios (HR) or OR) and the corresponding 95\% CI, or sufficient information for their calculation. In all, three authors (C. B., D. E. and V. B.) independently screened the search results against these criteria to identify eligible articles, and checked the reference list of the publications retrieved to identify additional publications. Discrepancies were discussed and resolved in consensus. If additional data were requested from the identified publications, contact with authors was realised.

\section{Study selection}

The flow chart for the selection procedure, undertaken by our group in December 2017, is shown in Fig. 1. The corresponding

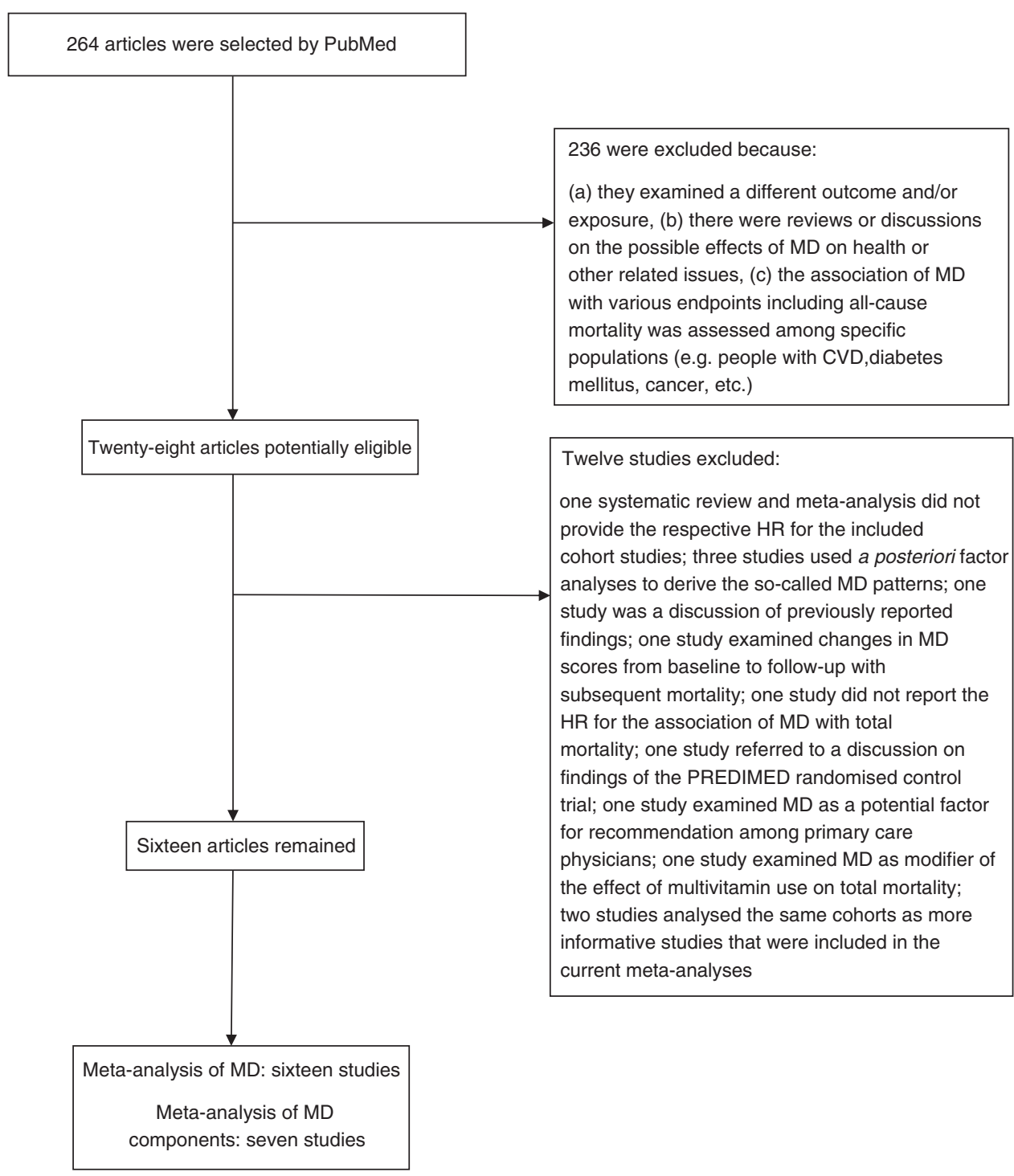

Fig. 1. Selection strategy to identify studies that were published from 1 July 2013 to 31 December 2017 and were included in the meta-analysis of the Mediterranean diet (MD) and overall mortality. HR, hazard ratio; PREDIMED, PREvención con Dleta MEDiterránea. 
information for studies published up to June 2013 has been published $^{(4)}$. This search identified 264 articles, with all abstracts published in English, included in the PubMed database, which were fully examined. From these, 236 were excluded because (a) they examined a different outcome and/ or exposure, (b) there were reviews or discussions on the possible effects of the MedDiet on health or other related issues and (c) the association of the MedDiet with various end points including all-cause mortality was assessed among specific populations (e.g. people with CVD, diabetes mellitus, cancer and so on). From the remaining twenty-eight papers, the following were further excluded: one systematic review and metaanalysis evaluating the health effects of the MedDiet with various health outcomes ${ }^{(11)}$ because it did not provide the respective $\mathrm{HR}$ for the included cohort studies; three studies ${ }^{(12-14)}$ because the so-called MedDiet patterns were derived from $a$ posteriori factor analyses and not a priori; one study ${ }^{(15)}$ because it was a discussion of previously reported findings that are included in the current meta-analysis ${ }^{(16)}$; one study ${ }^{(17)}$ because the association with all-cause mortality was examined for changes in MedDiet scores from baseline to follow-up, and therefore could not match the rest of the studies that reported associations of baseline MedDiet with subsequent mortality; one study because it did not report the HR for the association of the MedDiet with total mortality but only for CVD incidence/ mortality ${ }^{(18)}$; the study of Guasch-Ferre et al. ${ }^{(19)}$ because it referred to a discussion on findings of the PREvención con DIeta MEDiterránea (PREDIMED) RCT; the study by Ebell \& $\mathrm{Grad}^{(20)}$ in which the MedDiet was examined as a potential factor for recommendation among primary care physicians; and the study by Rautiainen et $a l .{ }^{(21)}$ in which the MedDiet was examined as a modifier of the effect of multivitamin use on total mortality (among other outcomes) in the Physicians' Health Study II RCT. Furthermore, the study by Harmon et al. ${ }^{(22)}$ was also excluded as the subsequent study by Shvetsov et al. ${ }^{(23)}$ used the same study population and was more informative. Similarly, the study by Liese et $a l .{ }^{(24)}$ was excluded because the HR of the MedDiet with all-cause mortality were only graphically displayed and it was not possible to derive the actual values - nonetheless, the cohorts analysed in the indicated publication were the same as in three other studies $^{(23,25,26)}$ that reported the respective HR and were included in our meta-analysis.

A total of sixteen articles were finally identified through the above process. One further study ${ }^{(27)}$ was identified upon examination of the references of these studies; this was an update of the study conducted by Trichopoulou et al. ${ }^{(28)}$ using the European Prospective Investigation into Cancer and Nutrition (EPIC) multi-centre cohort. However, as the study by Trichopoulou et $a l .{ }^{(28)}$ contained more information relevant to the current meta-analysis (RR for each of the included cohorts and RR for each of the components of the MedDiet), we decided to keep the earlier study. Nonetheless, the summary RR of the overall meta-analysis remained the same when the most recent study by Lassale et al. ${ }^{(27)}$ was included instead of the Trichopoulou et al. ${ }^{(28)}$ study.

The studies analysed by Sofi et $a l^{(4)}$ were also included in the current meta-analysis, except for the study by Martínez-González et $a l^{(8)}$, as the most recent publication by Alvarez-Alvarez et $a l^{(29)}$ used the same cohort and was more informative. Therefore, thirty articles were finally included in the meta-analyses of the MedDiet with all-cause mortality: fourteen published before $^{(7,16,28,30-40)}$ and sixteen published after June 2013 (up to 31 December 2017) $)^{(9,10,23,25,26,29,41-50)}$. Detailed characteristics of these studies are shown in Table 1. In eighteen of these publications, RR of all-cause mortality associated with at least one of the components of the MedDiet were reported.

\section{Data extraction}

For each study, data were extracted on study design, country, duration of follow-up, enrolment period, number of subjects or person(time)-at-risk, number of deceased, age of the study population, estimates of RR (e.g. HR) and their corresponding $95 \% \mathrm{CI}$, construction rule of adherence to the MedDiet and range of MDS and covariates adjusted for in the analysis. If a study used more than one MedDiet index to measure the adherence to the MedDiet ${ }^{(9,23,29,34,36,40)}$ the one more closely resembling the first, original MedDiet index that appeared in the literature ${ }^{(30,51)}$ was used. From each study, we selected RR estimates adjusted for the largest number of potential confounders.

The MedDiet was assessed in the vast majority of the studies through FFQ (quantitative or semi-quantitative) enquiring usual dietary intakes over a period preceding enrolment (e.g. previous year). Dietary history ${ }^{(32,36,37)}$, dietary records or weighted food records ${ }^{(35,39,41,42)}$ and 24-h dietary recall ${ }^{(43)}$ were also used. Most FFQ had been validated for (at least some) dietary intakes of the MedDiet components.

RR for all-cause mortality were reported: (a) only for continuous increments of MDS (e.g. one or two units) in nine stu$\operatorname{dies}^{(7,9,16,30-32,40,42,50)}$, (b) only as a comparison of different categories of MDS in ten studies ${ }^{(23,25,26,29,34,39,41,47-49)}$ and (c) for both (a) and (b) in eleven studies ${ }^{(10,28,33,35-38,43-46)}$. Categories of MDS were defined arbitrarily or were based on percentiles.

\section{Statistical analysis}

Mediterranean diet score. To estimate more closely the association of the MedDiet with all-cause mortality, we considered separately studies that reported RR for contrasting categories of upper to lowest MDS (thus assuming a non-linear association) and studies that estimated RR for continuous increments of MDS (thus assuming a log linear shape of the association). For the former studies, RR for the highest $v$. lowest study-specific categories of MDS were analysed. For the latter studies, RR were re-estimated (if needed) to correspond to 1SD increment in MDS, in order to account for differences in the range of MDS values.

For studies that did not report SD for the MDS, we assumed the same SD as those reported in other studies that used the same MDS. In the study by Tong et al. ${ }^{(9)}$, the respective SD was obtained by personal communication with the authors. For the study of Prinelli et al. ${ }^{(44)}$, the SD of the MDS was obtained by the study of Georgousopoulou et al. ${ }^{(52)}$, which used the same score and reported its SD. 
Table 1. Main characteristics of prospective studies included in the meta-analyses reporting on the association of the Mediterranean diet (MedDiet) and of its components and overall mortality

\begin{tabular}{|c|c|c|c|c|c|c|c|c|c|c|}
\hline No. & $\begin{array}{l}\text { Study } \\
\text { reference/cohort } \\
\text { name }\end{array}$ & Location & $\begin{array}{l}\text { Enrolment/ } \\
\text { follow-up }\end{array}$ & Cohort size No. of & f deaths & MDS $^{*}$ construction & MDS range & $\begin{array}{l}\text { Covariates controlled for in the relative } \\
\text { risk estimate }\end{array}$ & Age range, sex & $\begin{array}{l}\text { Dietary assessment } \\
\text { method/validation }\end{array}$ \\
\hline 1 & $\begin{array}{l}\text { Trichopoulou et al. } \\
\text { (1995)†/NA }\end{array}$ & Greece & $\begin{array}{l}\text { 1988-1990/3-6 } \\
\text { years }\end{array}$ & 182 & 53 & $\begin{array}{l}\text { Components: } 8 \\
\text { High: vegetables/legumes/ } \\
\text { cereals/fruit and nuts/ } \\
\text { MUFA:SFA } \\
\text { Low: meat/dairy } \\
\text { Moderate: alcohol } \\
\text { Cut-off: sex-specific median } \\
\text { intakes }\end{array}$ & $0-8$ & Age, sex, current smoking status & $\begin{array}{l}>70 \text { years, men and } \\
\text { women }\end{array}$ & $\begin{array}{l}\text { Semi-quantitative FFQ } \\
\text { (190 items)/yes }\end{array}$ \\
\hline 2 & $\begin{array}{c}\text { Kouris-Blazos et al. } \\
(1999)+/ \mathrm{NA}\end{array}$ & Australia & $\begin{array}{l}\text { 1990-1992/46 } \\
\text { years }\end{array}$ & 330 & 38 & $\begin{array}{l}\text { Components: } 8 \\
\text { High: vegetables/legumes/ } \\
\text { cereals/fruit and nuts/ } \\
\text { MUFA:SFA } \\
\text { Low: meat/dairy } \\
\text { Moderate: alcohol } \\
\text { Cut-off: sex-specific median } \\
\text { intakes }\end{array}$ & $0-8$ & $\begin{array}{l}\text { Age, sex, current smoking status, } \\
\text { ethnic origin }\end{array}$ & $\begin{array}{l}\geq 70 \text { years, men and } \\
\text { women }\end{array}$ & $\begin{array}{l}\text { Semi-quantitative FFQ } \\
\text { (250 items)/yes }\end{array}$ \\
\hline 3 & $\begin{array}{l}\text { Lasheras et al. } \\
(2000)+/ N A\end{array}$ & Spain & $1989 / \geq 9$ years & 161 & 96 & $\begin{array}{l}\text { Components: } 8 \\
\text { High: vegetables/legumes/ } \\
\text { cereals/fruit and nuts/ } \\
\text { MUFA:SFA } \\
\text { Low: meat/dairy } \\
\text { Moderate: alcohol } \\
\text { Cut-off: sex- and age-specific } \\
\text { median intakes }\end{array}$ & $0-8$ & $\begin{array}{l}\text { Age, sex, albumin concentration, self- } \\
\text { assessment of health, physical } \\
\text { activity, BMI, dieting in response to } \\
\text { chronic conditions, components of } \\
\text { the score were adjusted to } \\
10460 \mathrm{~kJ} \text { for men, } 8368 \mathrm{~kJ} \text { for } \\
\text { women }\end{array}$ & $\begin{array}{l}\text { 65-95 years, men } \\
\text { and women }\end{array}$ & $\begin{array}{l}\text { Semi-quantitative } \\
\text { FFQ/no }\end{array}$ \\
\hline 4 & $\begin{array}{l}\text { Knoops et al. } \\
(2004)+/ \text { HALE } \\
\text { study }\end{array}$ & $\begin{array}{l}\text { Eleven European } \\
\text { countries }\end{array}$ & $\begin{array}{l}\text { 1988-2000/10 } \\
\text { years }\end{array}$ & 2339 & 935 & $\begin{array}{l}\text { Components: } 8 \\
\text { High: vegetables and } \\
\text { potatoes/legumes/grains/ } \\
\text { fruit/nuts and seeds/fish/ } \\
\text { MUFA:SFA } \\
\text { Low: meat/dairy } \\
\text { Moderate: alcohol } \\
\text { Cut-off: sex-specific median } \\
\text { intakes }\end{array}$ & $0-8$ & $\begin{array}{l}\text { Age, sex, years of education, BMI, } \\
\text { study, moderate alcohol } \\
\text { consumption, physical activity, non- } \\
\text { smoking, other dietary factors, } \\
\text { components of the score were } \\
\text { adjusted to } 10460 \mathrm{~kJ} \text { for men, } \\
8368 \mathrm{~kJ} \text { for women }\end{array}$ & $\begin{array}{l}\text { 70-90 years, men } \\
\text { and women }\end{array}$ & Diet history method/yes \\
\hline 5 & $\begin{array}{l}\text { Trichopoulou et al. } \\
(2005) \dagger / \text { EPIC- } \\
\text { Elderly }\end{array}$ & $\begin{array}{l}\text { Nine European } \\
\text { countries }\end{array}$ & $\begin{array}{l}\text { 1992-2000/ } \\
\text { (median } \\
89 \text { months) }\end{array}$ & 74607 & 4047 & $\begin{array}{l}\text { Components: } 9 \\
\text { High: vegetables/legumes/ } \\
\text { cereals/fruit and nuts/fish/ } \\
\text { MUFA + PUFA:SFA } \\
\text { Low: meat/dairy } \\
\text { Moderate: alcohol } \\
\text { Cut-off: sex-specific median } \\
\text { intakes }\end{array}$ & $0-9$ & $\begin{array}{l}\text { Sex, age, diabetes at baseline, } \\
\text { waist:hip ratio, BMI, education, } \\
\text { smoking, physical activity at work, } \\
\text { physical activity at leisure, } \\
\text { consumption of potatoes, } \\
\text { consumption of eggs, consumption } \\
\text { of sugar and confectionery, total } \\
\text { energy intake }\end{array}$ & $\begin{array}{l}\geq 60 \text { years, men and } \\
\text { women }\end{array}$ & $\begin{array}{l}\text { FFQ or dietary records } \\
\text { for } 7 \text { or } 14 \mathrm{~d} / \text { yes }\end{array}$ \\
\hline 6 & $\begin{array}{l}\text { Lagiou et al. (2006)†/ } \\
\text { NA }\end{array}$ & Sweden & $\begin{array}{r}1991-1992 / \\
12 \text { years }\end{array}$ & 42237 & 572 & $\begin{array}{l}\text { Components: } 9 \\
\text { High: vegetables/legumes/ } \\
\text { cereals/fruit and nuts/fish/ } \\
\text { MUFA:SFA } \\
\text { Low: meat/milk and dairy } \\
\text { Moderate: alcohol } \\
\text { Cut-off: median intakes }\end{array}$ & $0-9$ & $\begin{array}{l}\text { Age, height, BMI, smoking status, } \\
\text { physical activity, education, energy } \\
\text { intake, potato, egg, } \\
\text { polyunsaturated lipid, sweet and } \\
\text { sugar and non-alcoholic beverage } \\
\text { intake }\end{array}$ & 30-49 years, women & FFQ (80 items)/yes \\
\hline 7 & $\begin{array}{l}\text { Mitrou et al. (2007)/ } \\
\text { NIH-AARP Diet } \\
\text { and Health study }\end{array}$ & $\begin{array}{l}\text { Six states and two } \\
\text { metropolitan } \\
\text { areas, USA }\end{array}$ & $\begin{array}{l}1995-1996 \\
/ 10 \text { years }\end{array}$ & $\begin{array}{l}\text { M: } 214284 \\
\text { (18 126) } \\
\text { W: } 166012(9673)\end{array}$ & & $\begin{array}{l}\text { Components: } 9 \\
\text { High: vegetables/legumes/ } \\
\text { grains/fruit and nuts/fish/ } \\
\text { MUFA:SFA } \\
\text { Low: meat/dairy } \\
\text { Moderate: alcohol } \\
\text { Cut-off: sex-specific median } \\
\text { intakes }\end{array}$ & $0-9$ & $\begin{array}{l}\text { Age, total energy intake, smoking, } \\
\text { education, BMI, physical activity, } \\
\text { race, marital status, menopausal } \\
\text { hormone therapy }\end{array}$ & $\begin{array}{l}\text { 50-71 years, men } \\
\text { and women }\end{array}$ & FFQ (124 items)/yes \\
\hline
\end{tabular}




\section{N British Journal of Nutrition}

Table 1. Continued

\begin{tabular}{|c|c|c|c|c|c|c|c|c|c|c|}
\hline No. & $\begin{array}{l}\text { Study } \\
\text { reference/cohort } \\
\text { name }\end{array}$ & Location & $\begin{array}{l}\text { Enrolment/ } \\
\text { follow-up }\end{array}$ & Cohort size No. of $\mathrm{c}$ & f deaths & MDS $^{*}$ construction & MDS range & $\begin{array}{l}\text { Covariates controlled for in the relative } \\
\text { risk estimate }\end{array}$ & Age range, sex & $\begin{array}{l}\text { Dietary assessment } \\
\mathrm{method} / \mathrm{validation}\end{array}$ \\
\hline 8 & $\begin{array}{l}\text { Trichopoulou et al. } \\
\text { (2009)†/EPIC } \\
\text { study }\end{array}$ & Greece & $\begin{array}{l}1994-1999 / 8.5 \\
\text { years (mean) }\end{array}$ & 23349 & 1075 & $\begin{array}{l}\text { Components: } 9 \\
\text { High: vegetables/ } \\
\text { legumes/cereals/ } \\
\text { fruit and nuts//ish/ } \\
\text { MUFA:SFA } \\
\text { Low: meat/dairy } \\
\text { Moderate: alcohol } \\
\text { Cut-off: sex-specific median } \\
\text { intakes }\end{array}$ & $0-9$ & $\begin{array}{l}\text { sex, age, education, smoking status, } \\
\text { BMl, MET score, total energy } \\
\text { intake }\end{array}$ & $\begin{array}{l}20-86 \text { years, men } \\
\text { and women }\end{array}$ & FFQ (150 items)/yes \\
\hline 9 & $\begin{array}{l}\text { Sjögren et al., 2010/ } \\
\text { NA }\end{array}$ & Sweden & $\begin{array}{l}\text { Beginning of } \\
\text { '90s/10.2 } \\
\text { years median }\end{array}$ & 924 & 215 & $\begin{array}{l}\text { Components: } 8 \\
\text { High: vegetables and } \\
\text { legumes/cereals/fruit/fish/ } \\
\text { PUFA:SFA } \\
\text { Low: meat/dairy } \\
\text { Moderate: alcohol } \\
\text { Cut-off: sex-specific, energy- } \\
\text { adjusted median intakes }\end{array}$ & $0-8$ & $\begin{array}{l}\text { Energy intake, smoking, social class, } \\
\text { type } 2 \text { diabetes, the metabolic } \\
\text { syndrome, lipid-lowering treatment, } \\
\text { waist circumference, diastolic blood } \\
\text { pressure, insulin, C-reactive protein }\end{array}$ & $71 \pm 1$ years, men & 7-d dietary records/yes \\
\hline 10 & $\begin{array}{l}\text { Tognon et al. } \\
\quad(2011) \dagger \dagger \\
\text { gerontological } \\
\text { and geriatric } \\
\text { population studies } \\
\text { in Gotenburg }\end{array}$ & Sweden & $\begin{array}{l}1971,1981 \\
\quad 1992,2000 / \\
2009\end{array}$ & 1037 & 630 & $\begin{array}{l}\text { Components: } 9 \\
\text { High: vegetables and } \\
\text { potatoes/legumes, nuts, } \\
\text { seeds/wholegrain cereals/ } \\
\text { fruit and fruit juices/fish/ } \\
\text { MUFA+PUFA:SFA/ } \\
\text { alcohol } \\
\text { Low: meat and eggs/ dairy } \\
\text { Cut-off: sex-specific median } \\
\text { intakes }\end{array}$ & $0-9$ & $\begin{array}{l}\text { Sex, BMI, waist circumference, } \\
\text { physical activity, marital status, } \\
\text { smoking, birth cohort, education }\end{array}$ & $\begin{array}{l}70 \text { years, men and } \\
\text { women }\end{array}$ & Diet history/yes \\
\hline 11 & $\begin{array}{l}\text { Buckland et al. } \\
(2011)+/ \text { EPIC } \\
\text { Spain }\end{array}$ & Spain & $\begin{array}{l}1992-1996 / \\
2006-2009\end{array}$ & 40622 & 1855 & $\begin{array}{l}\text { Components: } 9 \\
\text { High: vegetables/fruit, nuts } \\
\text { and seeds/legumes/fish/ } \\
\text { olive oil and cereals } \\
\text { Low: total meat/dairy } \\
\text { Moderate: alcohol } \\
\text { Cut-off: tertiles of intake }\end{array}$ & $0-18$ & $\begin{array}{l}\text { BMI, waist circumference, education, } \\
\text { physical activity, smoking status } \\
\text { and intensity, total energy intake, } \\
\text { age, sex, centre }\end{array}$ & $\begin{array}{l}\text { 29-69 years, men } \\
\text { and women }\end{array}$ & $\begin{array}{l}\text { Diet history } \\
\quad \text { questionnaire } \\
\text { (computerised)/yes }\end{array}$ \\
\hline 12 & $\begin{array}{l}\text { van den Brandt et al. } \\
(2011)+\text { /NLCS } \\
\text { study }\end{array}$ & Netherlands & 1986/1996 & $\begin{array}{l}\text { M: } 58279 \\
(6329) \\
\text { W: } \\
62573 \\
(3362)\end{array}$ & & $\begin{array}{l}\text { Components: } 9 \\
\text { High: vegetables/legumes/ } \\
\text { fruit/nuts/whole grains/fish/ } \\
\text { MUFA:SFA } \\
\text { Low: red and processed meat } \\
\text { Moderate: alcohol } \\
\text { Cut-off: sex-specific median }\end{array}$ & $0-9$ & $\begin{array}{l}\text { Age, cigarette smoking, number of } \\
\text { cigarettes/d, years smoking, BMI, } \\
\text { non-occupational physical activity, } \\
\text { history of hypertension, highest } \\
\text { level of education, energy intake }\end{array}$ & $\begin{array}{l}55-69 \text { years, men } \\
\text { and women }\end{array}$ & $\begin{array}{l}\text { Semi-quantitative FFQ } \\
\text { (150 items)/yes }\end{array}$ \\
\hline 13 & $\begin{array}{l}\text { McNaugton et al. } \\
\text { (2012)/British Diet } \\
\text { and Nutrition } \\
\text { Survey }\end{array}$ & Great Britain & $\begin{array}{l}1994-1995 / \\
2008\end{array}$ & 972 & 654 & $\begin{array}{l}\text { Components: } 9 \\
\text { High: vegetables/legumes/ } \\
\text { cereals/fruit and nuts/fish/ } \\
\text { MUFA:SFA } \\
\text { Low: meat/dairy } \\
\text { Moderate: alcohol } \\
\text { Cut-off: sex-specific medians }\end{array}$ & $0-9$ & $\begin{array}{l}\text { Age, sex, energy intake, social class, } \\
\text { region, smoking, physical activity } \\
\text { and BMI }\end{array}$ & $\begin{array}{l}\geq 65 \text { years, men and } \\
\text { women }\end{array}$ & 4-d weighted food record \\
\hline 14 & $\begin{array}{l}\text { Tognon et al. } \\
(2012) \dagger / \text { IP } \\
\text { cohort }\end{array}$ & Sweden & $\begin{array}{l}\text { 1990-2008/18 } \\
\text { years } \\
\text { average }\end{array}$ & $\begin{array}{c}\text { M: } 37546 \\
(1453) \\
W: 39605 \\
(923)\end{array}$ & & $\begin{array}{l}\text { Components: } 8 \\
\text { High: vegetables and } \\
\text { potatoes/fruit and juices/ } \\
\text { whole-grain cereals/fish/ } \\
\text { MUFA+PUFA:SFA/ } \\
\text { alcohol } \\
\text { Low: meat/dairy } \\
\text { Cut-off: sex-specific, energy- } \\
\text { adjusted, FFQ version } \\
\text { specific, medians }\end{array}$ & $0-8$ & $\begin{array}{l}\text { Age, obesity, physical activity, } \\
\text { smoking status, education }\end{array}$ & $30-60$ years & $\begin{array}{l}\text { FFQ }(84 \text { items or } 65 \\
\text { items }) / \text { yes }\end{array}$ \\
\hline
\end{tabular}


Table 1. Continued

\begin{tabular}{|c|c|c|c|c|c|c|c|c|c|c|}
\hline No. & $\begin{array}{l}\text { Study } \\
\text { reference/cohort } \\
\text { name }\end{array}$ & Location & $\begin{array}{l}\text { Enrolment/ } \\
\text { follow-up }\end{array}$ & Cohort size I & o. of deaths & MDS $^{*}$ construction & MDS range & $\begin{array}{l}\text { Covariates controlled for in the relative } \\
\text { risk estimate }\end{array}$ & Age range, sex & $\begin{array}{l}\text { Dietary assessment } \\
\text { method/validation }\end{array}$ \\
\hline 15 & $\begin{array}{l}\text { Cuenca-García et al. } \\
\text { (2014)/Aerobics } \\
\text { Center } \\
\text { Longitudinal } \\
\text { Study }\end{array}$ & USA & $\begin{array}{r}1987-1999 / 11.6 \\
\text { years (mean) }\end{array}$ & 12449 & 358 & $\begin{array}{l}\text { Components: } 9 \\
\text { High: vegetables/legumes/ } \\
\text { cereals/fruit-nuts/fish/ } \\
\text { MUFA:SFA } \\
\text { Low: meat/dairy } \\
\text { Moderate: alcohol } \\
\text { Cut-off: sex-specific median } \\
\text { intakes }\end{array}$ & $0-9$ & $\begin{array}{l}\text { Age, sex, energy intake, baseline } \\
\text { examination year, physical activity, } \\
\text { smoking, alcohol intake, abnormal } \\
\text { electrocardiogram, parental history } \\
\text { of CVD, cardiorespiratory fitness }\end{array}$ & $\begin{array}{l}20-84 \text { years, men } \\
\text { and women }\end{array}$ & 3-d diet record \\
\hline 16 & $\begin{array}{l}\text { Tognon et al. } \\
(2014) \dagger / \text { Danish } \\
\text { MONICA }\end{array}$ & Denmark & $\begin{array}{l}\text { 1982-1983/14 } \\
\text { years } \\
\text { average }\end{array}$ & 1348 & 553 & $\begin{array}{l}\text { Components: } 8 \\
\text { High: vegetables/cereal } \\
\text { grains/fruitffish/ } \\
\text { MUFA+PUFA:SFA/ } \\
\text { alcohol } \\
\text { Low: meat and meat products } \\
\text { and eggs/dairy } \\
\text { Cut-off: sex-specific, energy } \\
\text { adjusted median intakes }\end{array}$ & $0-8$ & $\begin{array}{l}\text { Sex, BMI, education, physical activity } \\
\text { and cigarette smoking }\end{array}$ & $\begin{array}{l}\text { 30-60 years, men } \\
\text { and women }\end{array}$ & $\begin{array}{l}\text { Weighted } 7-d \text { food } \\
\text { record/yes }\end{array}$ \\
\hline 17 & $\begin{array}{l}\text { George et al. (2014)/ } \\
\text { Women's Health } \\
\text { Initiative } \\
\text { Observational } \\
\text { Study }\end{array}$ & USA & $\begin{array}{r}1993-1998 / 12 \cdot 9 \\
\text { years median }\end{array}$ & 63805 & 5692 & $\begin{array}{l}\text { Components: } 9 \\
\text { High: vegetables/legumes/ } \\
\text { whole grains/fruit/nuts and } \\
\text { seeds/fish/MUFF:SFA } \\
\text { Low: red and processed meat } \\
\text { Moderate: alcohol } \\
\text { Cut-off: sex-specific median } \\
\text { intakes }\end{array}$ & $0-9$ & $\begin{array}{l}\text { Age, energy intake, ethnicity, } \\
\text { education, marital status, smoking, } \\
\text { physical activity, BMI, } \\
\text { postmenopausal hormone } \\
\text { replacement therapy, diabetes }\end{array}$ & 50-79 years, women & FFQ (122 items)/yes \\
\hline 18 & $\begin{array}{l}\text { Reedy et al. (2014)/ } \\
\text { NIH-AARP Diet } \\
\text { and Health study }\end{array}$ & $\begin{array}{l}\text { Six states and two } \\
\text { metropolitan } \\
\text { areas, USA }\end{array}$ & $\begin{array}{l}\text { 1995-1996/15 } \\
\text { years }\end{array}$ & 424662 & 86419 & $\begin{array}{l}\text { Components: } 9 \\
\text { High: vegetables/legumes/ } \\
\text { whole grains/fruit/nuts/fish/ } \\
\text { MUFF:SFA } \\
\text { Low: red and processed meat } \\
\text { Moderate: alcohol } \\
\text { Cut-off: sex-specific median } \\
\text { intakes }\end{array}$ & $0-9$ & $\begin{array}{l}\text { Age, ethnicity, education, BMl, } \\
\text { smoking, vigorous physical activity, } \\
\text { energy intake, marital status and } \\
\text { diabetes. }\end{array}$ & $\begin{array}{l}\text { 50-71 years, men } \\
\text { and women }\end{array}$ & FFQ (124 items)/yes \\
\hline 19 & $\begin{array}{l}\text { Vormund et al. } \\
(2015) \dagger / \text { NRP1A } \\
\text { and MONICA }\end{array}$ & Switzerland & $\begin{array}{c}1977-1993 / 21 \cdot 4 \\
\text { years mean }\end{array}$ & 17861 & 3953 & $\begin{array}{l}\text { Components: } 9 \\
\text { High: salad/vegetables/fruit/ } \\
\text { whole grains/fish/MUFA/ } \\
\text { white meat/dairy/alcohol } \\
\text { Low: red or processed meat } \\
\text { Cut-offs: not applied }\end{array}$ & $0-9$ & $\begin{array}{l}\text { Age, sex, survey wave, marital status, } \\
\text { smoking, BMl, language reason } \\
\text { and nationality }\end{array}$ & $\begin{array}{l}45.2 \text { years (mean), } \\
\text { men and women, }\end{array}$ & $\begin{array}{l}24 \mathrm{~h} \text {-recall checklist with } \\
\text { yes/no mentioned }\end{array}$ \\
\hline 20 & $\begin{array}{l}\text { Prinelli et al. (2015)†/ } \\
\text { NA }\end{array}$ & Italy & $\begin{array}{r}1991-1995 / 17 \cdot 4 \\
\text { years median }\end{array}$ & 974 & 193 & $\begin{array}{l}\text { Components: } 11 \\
\text { High: non-refined cereals/ } \\
\text { fruit/vegetables/legumes/ } \\
\text { potatoes/fish/olive oil } \\
\text { Low: meat and meat } \\
\text { products/poultry/full-fat } \\
\text { dairy } \\
\text { Moderate: alcohol } \\
\text { (score assigned 0-5 for } \\
\text { each component) } \\
\text { Cut-offs: frequency of weekly } \\
\text { consumption }\end{array}$ & $19-45$ & $\begin{array}{l}\text { Sex, age, education, BMl, time spent } \\
\text { watching TV, energy intake }\end{array}$ & $\begin{array}{l}40-74 \text { years, men } \\
\text { and women }\end{array}$ & $\begin{array}{l}\text { Quantitative FFQ ( } 158 \\
\text { items)/not mentioned }\end{array}$ \\
\hline 21 & $\begin{array}{l}\text { Bellavia et al. (2016)/ } \\
\text { COSM and SMC }\end{array}$ & Sweden & $\begin{array}{l}\text { SMC: } 1987- \\
1990 / 15 \\
\text { years } \\
\text { COSM: } \\
1997 / 15 \\
\text { years }\end{array}$ & 71333 & 14697 & $\begin{array}{l}\text { Components: } 8 \\
\text { High: vegetables and fruit/ } \\
\text { legumes and nuts/non- } \\
\text { refined/high-fibre grains/ } \\
\text { fermented dairy products/ } \\
\text { fish/use of olive oil or } \\
\text { rapeseed oil } \\
\text { Low: red meat } \\
\text { Moderate: alcohol }\end{array}$ & $0-8$ & $\begin{array}{l}\text { Age, sex, BMI, diabetes, physical } \\
\text { activity, smoking, education }\end{array}$ & $\begin{array}{l}45-79 \text { years men, } \\
48-83 \text { years } \\
\text { women }\end{array}$ & FFQ (96 items)/yes \\
\hline
\end{tabular}


Table 1. Continued

\begin{tabular}{|c|c|c|c|c|c|c|c|c|c|c|}
\hline No. & $\begin{array}{l}\text { Study } \\
\text { reference/cohort } \\
\text { name }\end{array}$ & Location & $\begin{array}{l}\text { Enrolment/ } \\
\text { follow-up }\end{array}$ & Cohort size $\mathrm{N}$ & of deaths & MDS $^{*}$ construction & MDS range & $\begin{array}{l}\text { Covariates controlled for in the relative } \\
\text { risk estimate }\end{array}$ & Age range, sex & $\begin{array}{l}\text { Dietary assessment } \\
\text { method/validation }\end{array}$ \\
\hline 22 & $\begin{array}{l}\text { Shvetsov et al. } \\
\text { (2016)/Multiethnic } \\
\text { Cohort Study }\end{array}$ & $\begin{array}{l}\text { Hawaii or Los } \\
\text { Angeles area }\end{array}$ & $\begin{array}{l}1993-1996 / 13- \\
18 \text { years }\end{array}$ & 193527 & 51702 & $\begin{array}{l}\text { Cut-offs: not mentioned } \\
\text { Components: } 9 \\
\text { High: vegetables/fruit/ } \\
\text { legumes/fish/nuts/whole } \\
\text { grains/MUFA:SFA ratio } \\
\text { Medium: alcohol } \\
\text { Low: red and processed meat } \\
\text { Cut-off: sex-specific medians }\end{array}$ & $0-9$ & $\begin{array}{l}\text { Age, ethnicity, BMI, moderate-to- } \\
\text { rigorous physical activity, smoking, } \\
\text { education, marital status, hormone } \\
\text { replacement therapy, history of } \\
\text { diabetes, heart disease and } \\
\text { cancer, energy intake }\end{array}$ & $\begin{array}{l}45-75 \text { years, men } \\
\text { and women }\end{array}$ & $\begin{array}{l}\text { Quantitative FFQ } \\
\text { (182 items)/yes }\end{array}$ \\
\hline 23 & $\begin{array}{l}\text { Tong et al. (2016)/ } \\
\text { EPIC-Norfolk }\end{array}$ & UK & $\begin{array}{l}1993-1997 / \\
1998-2000\end{array}$ & 23902 & 5660 & $\begin{array}{l}\text { Components: } 9 \\
\text { High: vegetables/legumes/ } \\
\text { fruit and nuts/cereals/fish/ } \\
\text { olive oil } \\
\text { Low: total dairy products/and } \\
\text { meat products } \\
\text { Medium: alcohol } \\
\text { Cut-off: sex-specific tertiles }\end{array}$ & $0-9$ & $\begin{array}{l}\text { Age, sex, education, social class, } \\
\text { marital status, smoking, physical } \\
\text { activity, season of FFQ } \\
\text { assessment, BMl, waist } \\
\text { circumference, prevalent diabetes, } \\
\text { medication use, family history of } \\
\text { diseases }\end{array}$ & $\begin{array}{l}40-79 \text { years, men } \\
\text { and women }\end{array}$ & $\begin{array}{l}\text { Semi-quantitative FFQ } \\
\text { (130 items)/yes }\end{array}$ \\
\hline 24 & Bo et al. (2016)/NA & Italy & $\begin{array}{l}2001-2003 / 12 \\
\text { years (mean) }\end{array}$ & 1658 & 220 & $\begin{array}{l}\text { Components: } 9 \\
\text { High: vegetables/legumes/ } \\
\text { grains/fruit and nuts/fish/ } \\
\text { MUFA:SFA } \\
\text { Low: meat/dairy } \\
\text { Moderate: alcohol } \\
\text { Cut-off: energy-adjusted sex- } \\
\text { specific medians }\end{array}$ & $0-9$ & $\begin{array}{l}\text { Age, sex, BMI, smoking, systolic and } \\
\text { diastolic blood pressure, fasting } \\
\text { glucose, total and HDL-cholesterol, } \\
\text { education, MET, baseline CV score }\end{array}$ & $\begin{array}{l}\text { 45-64 years, men } \\
\text { and women }\end{array}$ & FFQ (148 items)/yes \\
\hline 25 & $\begin{array}{l}\text { Limongi et al. } \\
\text { (2017)t/ILSA } \\
\text { study }\end{array}$ & Italy & $\begin{array}{l}1992 / 7.1 \text { years } \\
(\text { mean) }\end{array}$ & 2265 & 665 & $\begin{array}{l}\text { Components: } 11 \\
\text { High: grains//ruit/vegetables/ } \\
\text { legumes/nuts and seeds/ } \\
\text { olive oil/fish } \\
\text { Low: red meat/processed } \\
\text { meat/sweets/eggs } \\
\text { Low to moderate: dairy } \\
\text { (score assigned } 0-4 \text { for } \\
\text { each component) } \\
\text { Cut-off: tertiles (numbers of } \\
\text { portions/d) }\end{array}$ & $0-44$ & $\begin{array}{l}\text { Age, sex, diabetes mellitus, } \\
\text { myocardial infarction, disability, } \\
\text { BMI } \geq 25 \mathrm{~kg} / \mathrm{m}^{2}, \text { MMSE score }<24 \text {, } \\
\text { GDS score } \geq 10\end{array}$ & $\begin{array}{l}\text { 65-84 years, men } \\
\text { and women }\end{array}$ & $\begin{array}{l}\text { Semi-quantitative FFQ } \\
\text { (49 items)/no }\end{array}$ \\
\hline 26 & $\begin{array}{l}\text { Stefler et al. (2017)†/ } \\
\text { HAPPIE study }\end{array}$ & $\begin{array}{l}\text { Czech republic, } \\
\text { Poland, Russian } \\
\text { Federation }\end{array}$ & $\begin{array}{l}2002-2005 / 7 \\
\text { years }\end{array}$ & 19333 & 1314 & $\begin{array}{l}\text { Components: } 9 \\
\text { High: fruit/vegetables/ } \\
\text { cereals/legumes/fish } \\
\text { Low: meat and meat } \\
\text { products/dairy products } \\
\text { Moderate: alcohol/olive oil } \\
\text { Cut-off: absolute values, } \\
\text { three-tier scoring system }\end{array}$ & $0-17$ & $\begin{array}{l}\text { Age, sex, cohort, smoking, education, } \\
\text { household amenities score, marital } \\
\text { status, energy intake, physical } \\
\text { activity, vitamin supplement intake }\end{array}$ & $\begin{array}{l}\text { Middle-aged men } \\
\text { and women }\end{array}$ & $\begin{array}{l}\text { Semi-quantitative FFQ } \\
\text { (136 Czech, } 148 \\
\text { Polish, } 147 \text { Russian, } \\
\text { items)/yes }\end{array}$ \\
\hline 27 & $\begin{array}{l}\text { Whalen et al. (2017)/ } \\
\text { REGARDS study }\end{array}$ & USA & $\begin{array}{c}2003-2007 / 6 \cdot 25 \\
\text { years mean }\end{array}$ & 21423 & 2513 & $\begin{array}{l}\text { Components: } 11 \\
\text { High: vegetables/fruit/lean } \\
\text { meats/fish/nuts/MUFA: } \\
\text { SFA } \\
\text { Low: red and processed } \\
\text { meat/Na } \\
\text { Moderate: dairy/grains and } \\
\text { starches } \\
\text { Cut-offs: quintile rank } \\
\text { (e.g. highest and lowest } \\
\text { quintiles scored } 5 \text { and } \\
1 \text { points, respectively) }\end{array}$ & $11-55$ & $\begin{array}{l}\text { Sex, race, total energy intake, BMI, } \\
\text { physical activity, smoking, annual } \\
\text { income, HRT }\end{array}$ & $\begin{array}{l}\geq 45 \text { years, men and } \\
\text { women }\end{array}$ & FFQ (109 items)/yes \\
\hline
\end{tabular}


Table 1. Continued

\begin{tabular}{|c|c|c|c|c|c|c|c|c|c|c|}
\hline No. & $\begin{array}{l}\text { Study } \\
\text { reference/cohort } \\
\text { name }\end{array}$ & Location & $\begin{array}{l}\text { Enrolment/ } \\
\text { follow-up }\end{array}$ & Cohort size No & of deaths & MDS $^{*}$ construction & MDS range & $\begin{array}{l}\text { Covariates controlled for in the relative } \\
\text { risk estimate }\end{array}$ & Age range, sex & $\begin{array}{l}\text { Dietary assessment } \\
\text { method/validation }\end{array}$ \\
\hline 28 & $\begin{array}{l}\text { Franzon et al. } \\
\text { (2017)/ULSAM } \\
\text { study }\end{array}$ & Sweden & $\begin{array}{l}\text { 1990-1991/14 } \\
\text { years }\end{array}$ & 1104 & 479 & $\begin{array}{l}\text { Components: } 8 \\
\text { High: vegetables and } \\
\text { legumes/fruit/cereals and } \\
\text { potatoes/fish/PUFA:SFA } \\
\text { Low: meat and meat } \\
\text { products/milk and dairy } \\
\text { products } \\
\text { Moderate: alcohol } \\
\text { Cut-off: not mentioned }\end{array}$ & $0-8$ & $\begin{array}{l}\text { Age, educational level, smoking } \\
\text { status, dietary pattern }\end{array}$ & $\begin{array}{l}71 \text { years (mean } \\
\text { age), (range } \\
69 \cdot 4-74 \cdot 1) \text {, men }\end{array}$ & 7-d food record \\
\hline 29 & $\begin{array}{l}\text { Alvarez-Alvarez et al. } \\
\quad(2018)+/ \text { SUN } \\
\text { project }\end{array}$ & Spain & $\begin{array}{l}\text { 1999/10.3 years } \\
\text { (mean) }\end{array}$ & 19467 & 305 & $\begin{array}{l}\text { Components: } 9 \\
\text { High: vegetables/legumes/ } \\
\text { cereals/fruit and nuts/fish/ } \\
\text { MUFA:SFA } \\
\text { Low: meat/dairy } \\
\text { Moderate: alcohol } \\
\text { Cut-off: sex-specific medians }\end{array}$ & $0-9$ & $\begin{array}{l}\text { Age, sex, BMI, energy intake, } \\
\text { year entering the cohort smoking } \\
\text { status, family history of CVD, } \\
\text { alcohol consumption, diabetes and } \\
\text { hypertension at baseline, self- } \\
\text { reported hypercholesterolaemia, } \\
\text { depression, education and } \\
\text { following a special diet at baseline }\end{array}$ & $\begin{array}{l}38.2 \text { years (mean } \\
\text { age), men and } \\
\text { women }\end{array}$ & $\begin{array}{l}\text { Semi-quantitative FFQ } \\
\text { (136 items)/yes }\end{array}$ \\
\hline 30 & $\begin{array}{l}\text { Shah et al. (2018)†/ } \\
\text { Cooper Center } \\
\text { Longitudinal } \\
\text { Study }\end{array}$ & USA, Texas & $\begin{array}{l}1987-1999 / 18 \\
\text { years (mean) }\end{array}$ & 11376 & 841 & $\begin{array}{l}\text { Components: } 9 \\
\text { High: vegetables/fruit and } \\
\text { nuts/MUFA:SFA/legumes/ } \\
\text { grains/fish } \\
\text { Low: dairy/meat } \\
\text { Moderate: alcohol } \\
\text { Cut-off: quintiles }\end{array}$ & $0-9$ & $\begin{array}{l}\text { Age, sex, smoking, energy intake, } \\
\text { physical activity, BMI, family history } \\
\text { CVD, baseline glucose, LDL, } \\
\text { systolic blood pressure }\end{array}$ & $\begin{array}{l}46.5 \text { years (mean), } \\
\text { men and women }\end{array}$ & 3-d dietary record \\
\hline
\end{tabular}

MDS, Mediterranean diet score; HALE, Healthy Ageing: a Longitudinal Study in Europe; EPIC, European Prospective Investigation into Cancer and Nutrition elderly study; NIH-AARP, National Institutes of Health - AARP (formerly known as the American Association of Retired Persons); M, men; W, women; MET, metabolic equivalents; NA, non-applicable; NLCS, Netherlands Cohort Study; VIP, Vasterbotten Intervention Program; MONICA, MONItoring trends and
determinants of CVD; NRP1A, National Research Program 1A; TV, television; COSM, Cohort of Swedish Men; SMC, Swedish Mammography Cohort; ILSA, Italian Longitudinal Study on Aging; MMSE, Mini Mental State Examination; GDS, Geriatric Depression Scale; HAPPIE, Health Alcohol and Psychosocial Factors in Eastern Europe; REGARDS: REasons for Geographic and Racial Differences in Stroke Study; HRT, hormone replacement therapy; ULSAM, Uppsala Longitudinal Study of Adult Men; SUN, Sequimenta Universidad de Navarra.

* The MedDiet indices from each study that were used in the current analysis are shown in this column, labelled for simplicity as MDS. The labels of these indices may be different in the original publications.

†Associations of (at least one) MedDiet components with mortality were reported in this study. 
Mediterranean diet components. Intakes of the following nine major MedDiet components were examined: vegetables, legumes, fruit/nuts, cereals, fish and shellfish, meat and meat products, milk and dairy products, alcohol and lipid ratio in any way this was defined (e.g. monounsaturated-to-saturated, mono + polyunsaturated-to-saturated and so on). We also considered RR reported for olive oil or wine intakes as these are the main sources of MUFA and alcohol intake, respectively, in the typical MedDiet.

Similarly to the analysis of MDS, mortality RR for each of the indicated food groups/nutrients were estimated in some studies for continuous increments in the intake of the MDS component or as comparisons of categories of the component intake. For continuous increments, we re-estimated mortality RR for one study-specific SD increment in order to account for differences in the intakes of the MedDiet components across different populations. Among studies reporting mortality RR for different categories of MedDiet components, the vast majority contrasted the above/below-median study-specific consumptions. For studies that used other categories of comparison, we considered in our meta-analysis the comparison that resembled more the above/below-median comparison.

Meta-analysis of Mediterranean diet score and Mediterranean diet score components. Summary RR were estimated by combining the study-specific RR (considered as explained above) using random-effects models to take into account the between-study heterogeneity ${ }^{(53)}$. Each study's In (RR) was weighted by the inverse of its variance plus the between-study variance component $\tau^{2}$ computed by the moment estimator ${ }^{(53)}$. Heterogeneity among studies was evaluated with $\chi^{2}$ test and $I^{2}$ statistic $^{(54,55)}$. Publication bias was evaluated through funnel plots ${ }^{(56)}$ and with the Egger's test ${ }^{(57)}$.

We also conducted meta-analyses in strata of geographical area (USA, Australia, Europe (Northern, Central/Eastern, Western, Mediterranean)), range of MDS scale $(0-9,>9)$, in order to account for the different spread of the distribution of the MedDiet indices used in each study, and year of publication (<2010, 2010-2014, >2014). RR estimated from the multi-centre EPIC study ${ }^{(28)}$ were considered both overall and cohort-specific in meta-analyses stratified by country.

Sensitivity analyses were also conducted by omitting one study at a time from the analyses and assessing its effect on the overall summary RR.

The significance threshold was set at $0 \cdot 05$, whereas all statistical analyses were conducted using $\mathrm{R}$ statistical software version 3.4.2.

\section{Results}

In Table 1 the main characteristics of the thirty articles (including a total of 225600 deaths) considering a quantitative relation between MDS and all-cause mortality are shown. Out of these, seven were conducted in North America, twenty-two in Europe and one in Australia. Most studies used a 9- or 10-scale MedDiet index, except for the studies conducted by Buckland et $a l .{ }^{(37)}$, Prinelli et al. ${ }^{(44)}$, Limongi et al. ${ }^{(47)}$, Stefler et al. ${ }^{(10)}$ and
Whalen et al. ${ }^{(48)}$, which used indices of broader ranges. Studies by Mitrou et al. ${ }^{(34)}$, Tognon et al. ${ }^{(36)}$, Tognon et al. ${ }^{(40)}$, Shvetsov et $a l .{ }^{(23)}$, Tong et al. ${ }^{(9)}$ and Alvarez-Alvarez et al. ${ }^{(29)}$ used more than one MedDiet index; from these the traditional MDS, the refined version of the modified Mediterranean diet score (refined mMDS), the alternate MDS without energy adjustment in each of the MedDiet components, the MDS based on cohortspecific medians (mMDS) and the MDS proposed by Trichopoulou et $a l^{(51)}$, respectively, were considered in the current meta-analyses, all labelled as MDS for reasons of simplicity.

\section{Highest v. lowest level of adherence to the Mediterranean diet in relation to all-cause mortality}

Fig. 2 shows the RR of all-cause mortality for the study-specific highest $v$. lowest MDS levels of adherence among the twentyone studies, which reported such associations. In most studies that used an MDS ranging from 0 to $8 / 9$, the highest/lowest level of adherence was 6-8 or 6-9 v. 0-2 or 0-3, respectively, except for the studies by Tognon et al. ${ }^{(36)}(6-9 v$. 0-5), van den Brandt $^{(38)}$ (5-9 v. 0-4) and Vormund et al. ${ }^{(43)}$ (6-9 v. <4). Buckland et al. ${ }^{(37)}$ contrasted 11-18 v. 0-6 scores of their 0-18 MDS scale, Prinelli et al. ${ }^{(44)}$ the Q3 $v$. Q1 of their 46-point MedDiet index, Limongi et al. ${ }^{(47)}$ the Q3: $>24 v$. Q1: $<20$ of their MDS ranging from 0 to 44, Stefler et al. ${ }^{(10)} 11-17 v$. 0-7 scores of a 0-18 point MDS and Whalen et al. ${ }^{(48)}$ the Q5: (36-50) $v$. Q1 (14-26) of their 0-55 MDS.

All studies reported inverse associations, which were statistically significant in sixteen of them. Only one study ${ }^{(41)}$ reported an increased (non significant) mortality risk (RR: 1·15, 95\% CI, $0 \cdot 81,1.64)$ with increased MDS.

The summary mortality ratio for the highest $v$. lowest MDS level indicates an inverse and significant association: RR 0.79 (95\% CI $0.77,0.81$ ). Heterogeneity was moderate overall $\left(I^{2}=42 \%\right.$; $P$-heterogeneity $\left.0 \cdot 02\right)$.

All-cause mortality in relation to 1SD increase in adherence to the Mediterranean diet

In Fig. 3, RR of all-cause mortality per one study-specific SD increase in MDS are shown for the twenty studies with available information. Study-specific SD ranged from $1 \cdot 4^{(28)}$ to $5^{(44)}$ units in MDS. Inverse associations, statistically significant in fifteen studies, were reported in all publications, except for the study by Lasheras et $a l^{(31)}$, which reported increased albeit not significant mortality among eighty-seven Spanish people $\geq 80$ years of age for a 1.7-unit increase in MDS.

The summary mortality RR per 1 sD MDS increment was 0.92 (95\% CI 0.90, 0.94), with moderate-to-high heterogeneity $\left(I^{2}=56 \%\right.$; $P$-heterogeneity $\left.<0 \cdot 01\right)$.

\section{Subgroup analysis}

Summary RR (95\% CI) for the highest $v$. lowest MDS level of adherence, as well as per 1SD MDS increment in strata of selected covariates, are shown in Table 2. As only two of the studies reporting RR for continuous increments of MDS were 


\begin{tabular}{lrr} 
Study & Deaths & \multicolumn{1}{c}{$n$} \\
Trichopoulou, 2005 & 4047 & 74607 \\
Lagiou (F), 2006 & 572 & 42237 \\
Mitrou (M), 2007 & 18126 & 214284 \\
Mitrou (F), 2007 & 9673 & 166012 \\
Sjögren, 2010 & 215 & 924 \\
Tognon, 2011 & 630 & 1037 \\
Buckland, 2011 & 1855 & 40622 \\
van den Brandt (M), 2011 & 6329 & 58279 \\
van den Brandt (F), 2011 & 3362 & 62573 \\
McNaugton, 2012 & 654 & 972 \\
Cuenca-Garcia, 2014 & 358 & 12449 \\
George, 2014 & 5692 & 63805 \\
Reedy (M), 2014 & 54871 & 242321 \\
Reedy (F), 2014 & 31548 & 182341 \\
Vormund, 2015 & 3953 & 17861 \\
Prinelli, 2015 & 193 & 974 \\
Bellavia, 2016 & 14697 & 71333 \\
Shvetson (M), 2016 & 27744 & 87338 \\
Shvetson (F), 2016 & 23958 & 106189 \\
Bo, 2016 & 220 & 1658 \\
Limongi, 2017 & 665 & 2265 \\
Stefler, 2017 & 1314 & 19333 \\
Whalen, 2017 & 2513 & 21423 \\
Franzon, 2017 & 479 & 1104 \\
Alvarez, 2018 & 305 & 19467 \\
& & \\
Fixed effect model & & \\
Random effects model & & \\
Heterogeneity: $I^{2}=42 \%, \tau^{2}=0.0011, \chi_{24}^{2}=41 \cdot 10(P=0.02)$
\end{tabular}

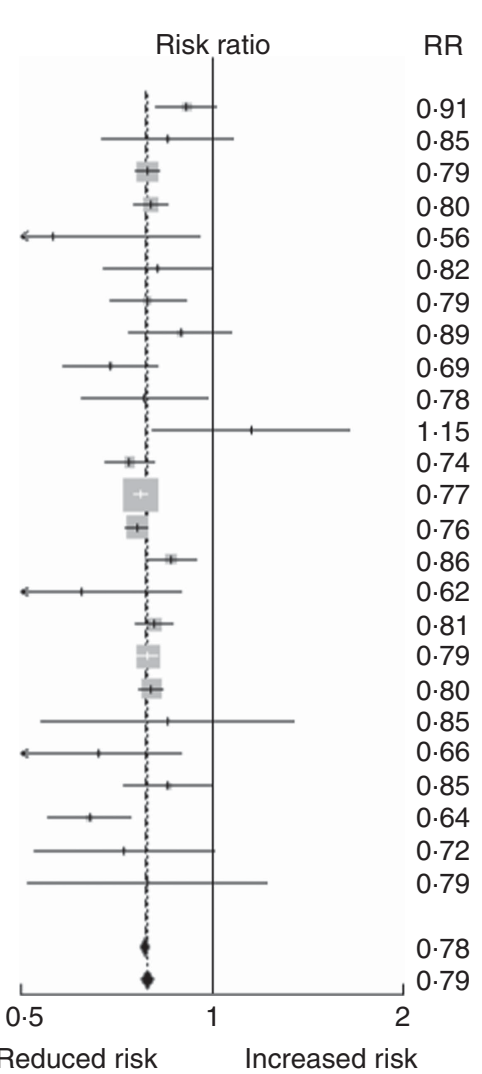

Weight Weight

$95 \% \mathrm{Cl} \quad(\%$, fixed) (\%, random)

$0.82,1.01$

$\begin{array}{ll}1.7 & 3.8\end{array}$

$0.67,1.08 \quad 0.4 \quad 1.0$

$0.76,0.83 \quad 10.4 \quad 10.1$

$0.75,0.85 \quad 5.2 \quad 7.6$

$0.33,0.96 \quad 0.1 \quad 0.2$

$0.67,1.00 \quad 0.5 \quad 1.4$

$0.69,0.91 \quad 1.1 \quad 2.6$

$0.74,1.07 \quad 0.6 \quad 1.6$

$\begin{array}{lll}0.58,0.82 & 0.7 & 1.8\end{array}$

$0.62,0.98 \quad 0.4 \quad 1.1$

$0.81,1.64 \quad 0.2 \quad 0.5$

$0.68,0.81 \quad 2.6 \quad 5.2$

$\begin{array}{lll}0.75,0.79 & 29.9 & 12.8\end{array}$

$0.73,0.79 \quad 12.9 \quad 10.8$

$0.78,0.94 \quad 2.3 \quad 4.8$

$0.43,0.89 \quad 0.2 \quad 0.4$

$0.76,0.87 \quad 4.3 \quad 6.9$

$0.76,0.82 \quad 14.0 \quad 11.0$

$0.77,0.84 \quad 10.4 \quad 10.1$

$0.54,1.34 \quad 0.1 \quad 0.3$

$\begin{array}{lll}0.49,0.89 & 0.2 & 0.6\end{array}$

$0.73,0.99 \quad 0.8 \quad 2.1$

$0.55,0.74 \quad 0.9 \quad 2.3$

$\begin{array}{lll}0.52,1.00 & 0.2 & 0.5\end{array}$

$0.51,1.22 \quad 0.1 \quad 0.3$

$\begin{array}{rrr}0.77,0.79 & 100.0 & - \\ 0.77,0.81 & - & 100.0\end{array}$

Reduced risk Increased risk

Fig. 2. Relative risks (RR) and $95 \% \mathrm{Cl}$ of mortality associated with the highest $v$. the lowest level of Mediterranean diet score from all studies included. The combined hazard ratio and $95 \% \mathrm{Cl}$ were calculated using the random-effects models. $\mathrm{F}$, female; $\mathrm{M}$, male.

conducted outside Europe (one in USA and one in Australia), stratified meta-analysis of RR per 1SD increment in MDS by geographical location was undertaken only among European studies.

RR of highest $v$. lowest MDS among studies undertaken in USA and Europe were 0.78 (95\% CI 0.76, 0.80) and $0.82(95 \%$ CI $0 \cdot 78,0 \cdot 88)$, respectively, with higher heterogeneity observed among the US $\left(I^{2}=55 \%, P\right.$-heterogeneity 0.02$)$ rather than European studies $\left(I^{2}=10 \%, P\right.$-heterogeneity $\left.0 \cdot 33\right)$. In analysis confined to European studies, stratification by geographical location revealed stronger summary RR among Mediterranean populations, and weakest ones among the fewer studies conducted in central/eastern Europe for both the highest/lowest or the per SD increment in MDS. Similar summary RR were estimated among studies with different spread of distribution of MDS - that is, those that used MDS scales of 0-9 or greater. Stratification by publication year revealed high consistency in RR after 2011, when the majority of the studies were published (Fig. 6(a) and (b)).

Heterogeneity was evident by country location. Across European studies heterogeneity was evident for RR corresponding to $1 \mathrm{SD}$ increment in MDS, but not for studies contrasting highest/lowest level of adherence to the MedDiet.

Sensitivity analysis and publication bias. In sensitivity analysis, the summary mortality RR in association with MDS adherence (highest $v$. lowest or per SD increment) did not change appreciably when each study was excluded in turn, and the RR was estimated from the remaining studies (mortality RR of highest $v$. lowest MDS ranged from 0.78 to 0.79 ; mortality RR per SD increment ranged from 0.92 to 0.93 ). The funnel plots of individual RR for the highest $v$. lowest MDS, and for the per SD MDS increment are shown in Figs. 4 and 5, respectively. There was no indication of publication bias for studies reporting RR for the highest $v$. lowest MDS adherence ( $P$-value $0 \cdot 67)$, but there was evidence for publication bias for studies reporting RR for $1 \mathrm{SD}$ increment in MDS $(P<0 \cdot 01)$, probably owing to the absence of studies reporting less precise RR showing increased mortality with continuous increments of MedDiet adherence. Nevertheless, when we used the trim-and-fill method including the missing studies, the estimated association between the perMDS increment and mortality did not change appreciably and retained its statistical significance.

All-cause mortality in relation to intakes of components of the Mediterranean diet. In all, eighteen studies reported associations of at least one component of the MedDiet with allcause mortality $(7,10,16,28-33,36-38,40,42-44,47,50)$. The studies by Knoops et $a l^{(32)}$ and Lagiou et $a l{ }^{(33)}$ reported RR only for alcohol intake; however, the latter study reported mortality RR contrasting high $v$. moderate ethanol consumption, which was incompatible with the contrasting categories of alcohol intake reported in the rest of the studies (mostly above/below median or moderate $v$. other). Therefore, that article was not included in 


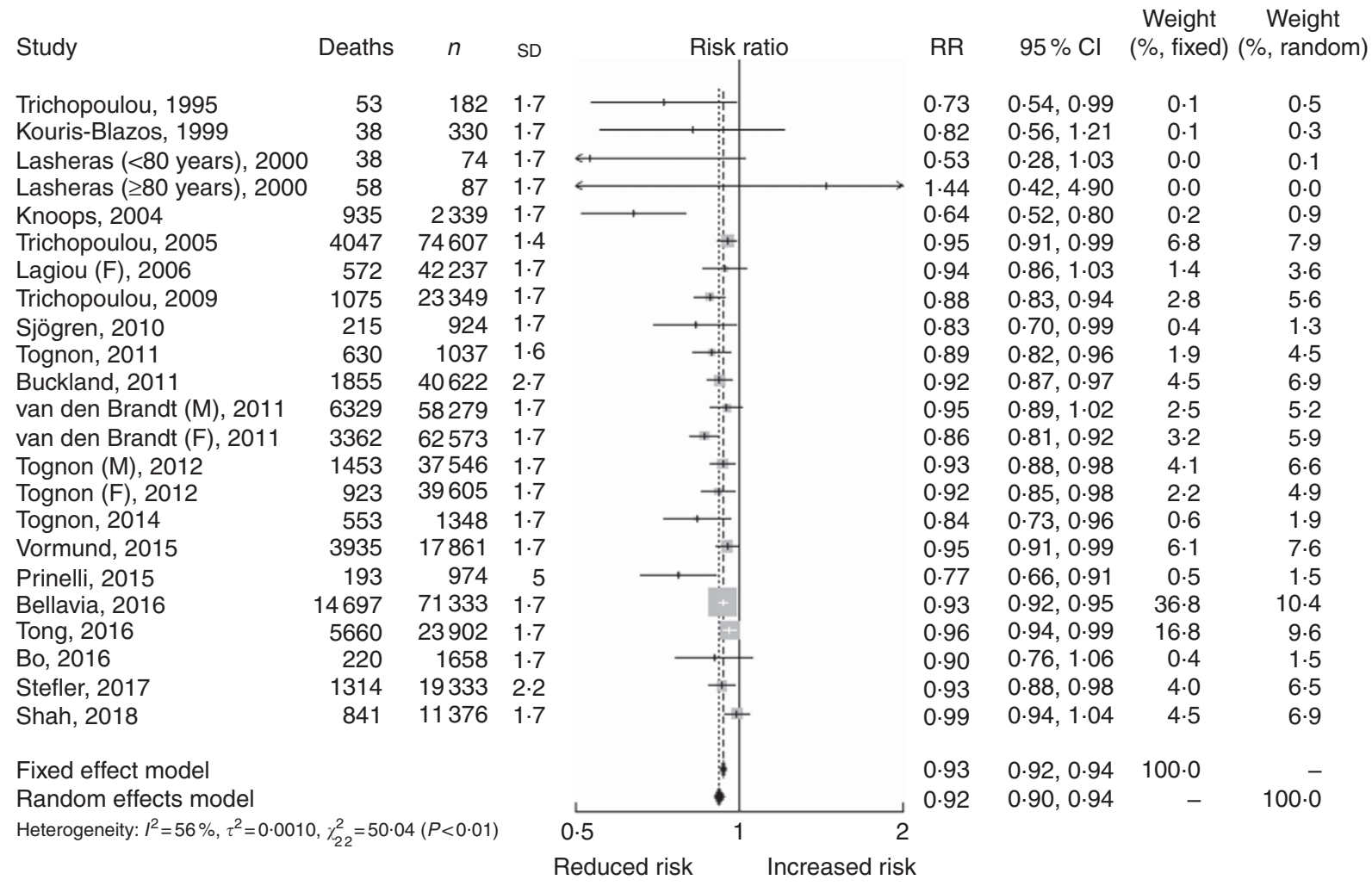

Fig. 3. Relative risks (RR) and $95 \% \mathrm{Cl}$ of mortality per one study-specific standard deviation increment in Mediterranean diet score from all studies included. The combined hazard ratio and $95 \% \mathrm{Cl}$ were calculated using the random-effects models. F, female; $\mathrm{M}$, male.

the meta-analysis of alcohol intake. Moreover, the study by Vormund et $a l .{ }^{(43)}$ reported mortality ratios contrasting consumers $v$. non-consumers for each of the MedDiet components; these categories of comparison were incompatible with those used in the rest of the studies (above/below median or per specific MDS increment). Thus, the indicated publication was also not included in the meta-analysis of MedDiet components in relation to mortality.

In Table 3, the summary RR of mortality (from random effects models) corresponding to the above/below median intake comparison, as well as to moderate intake $v$. other for alcohol consumption, are shown for each of the nine components of the MDS. Results from the meta-analyses of mortality RR per SD increment were in the same direction. Summary RR were based on different number of studies for each component, as some studies reported associations for a few components only.

Table 3 reveals those MedDiet components that showed a significant association with all-cause mortality either inverse (a) fruit/nuts, (b) vegetables, (c) alcohol (moderate $v$. other) - or positive - meat. With respect to the rest of the components, null associations were estimated for legumes and fish intake, weak/ minimal inverse associations for cereal and lipid ratio intakes, respectively, and a weak positive association for dairy intakes.

Olive oil in relation to mortality was investigated in four stu$\operatorname{dies}^{(10,37,44,47)}$, with $\mathrm{RR}$ reflecting the above $v$. below median intake comparison in all of them except for the study by Buckland $e$ t $a l .{ }^{(37)}$ who reported RR for the highest $(>24.7 \mathrm{~g} / \mathrm{d}) v$. lowest $(<13.7 \mathrm{~g} / \mathrm{d})$ consumption. No detailed information of the type of olive oil was given in these four studies. The summary RR of these studies was 0.97 (95\% CI 0.82, 1.15). Similarly, RR for wine intake in association with all-cause mortality were reported in two studies ${ }^{(40,42)}$ comparing the above/below median intakes. The summary RR based on these two studies was 0.87 (95\% CI $0.78,0.97)$.

In Fig. 7, the excess relative risk (ERR) associated with each of the MedDiet components is depicted as estimated from the summary RR. The ERR was highest for moderate alcohol intake $(-14 \%)$, followed by above-the-median intakes: fruit $(-12 \%)$, meat $(7 \%)$ and vegetable $(-6 \%)$ intakes, cereals $(-5 \%)$, dairy $(5 \%)$ and lipid ratio (-2\%) intakes. The ERR for fish and legume intake were close to zero.

\section{Discussion}

In the present meta-analyses of all published prospective investigations up to 31 December 2017, including thirty studies and 225600 deaths, we found an inverse, significant association between conformity to the MedDiet and all-cause mortality. We also estimated summary mortality ratios for each of the nine components of the MedDiet. In these analyses, relatively stronger and statistically significant associations were highlighted for moderate alcohol $(\mathrm{RR}=0 \cdot 86)$ consumption, as well as for above-the-median intakes of fruit $(\mathrm{RR}=0.88)$, meat $(\mathrm{RR}=1.07)$ and vegetables $(\mathrm{RR}=0.94)$.

Our findings are in agreement with recently published stu$\operatorname{dies}^{(4,6)}$. In the last meta-analysis of observational studies published up to June 2013, Sofi et al. ${ }^{(4)}$ estimated a summary RR of 
Table 2. Summary relative risks (RR) and $95 \% \mathrm{Cl}$ of all-cause mortality for the highest $v$. the lowest and per 1sD increment in the adherence to the Mediterranean diet (MedDiet) in strata of selected covariates

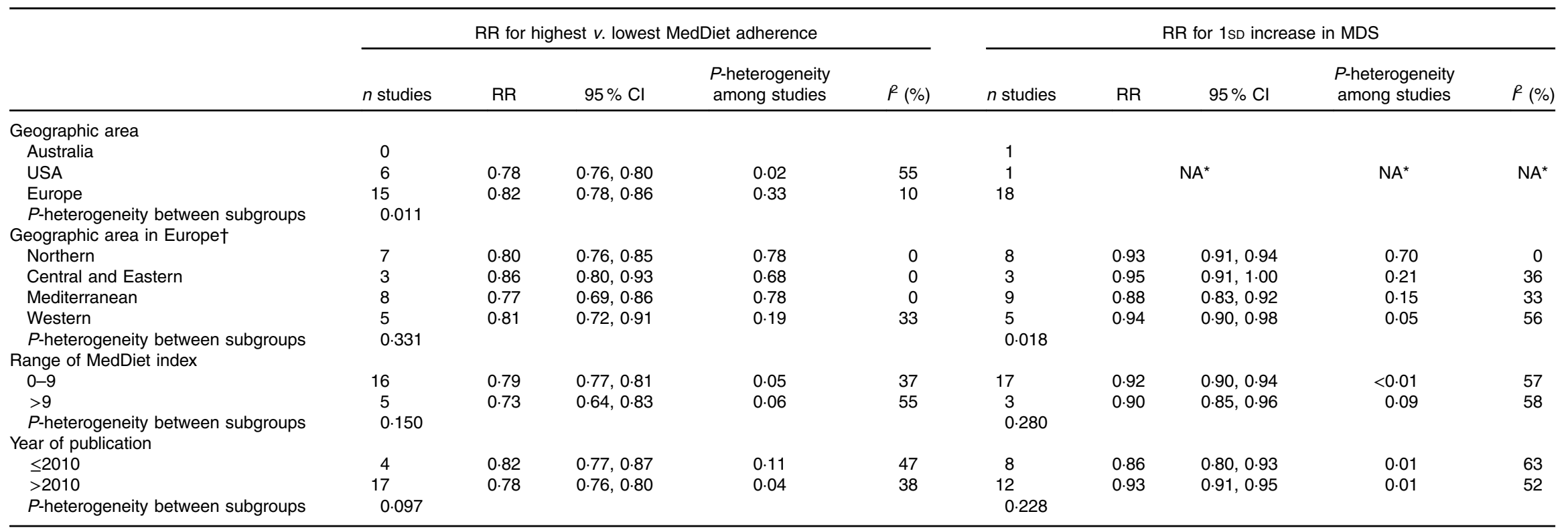

MDS, Mediterranean diet score; NA, non-applicable.

* Only two studies were undertaken outside Europe: one in the USA and one in Australia.

† In analysis among European countries, the nine cohort-specific RR reported in the study by Trichopoulou et al. (2005) were considered instead of the overall-cohorts RR, whereas the multi-centre European study by Knoops et al. (2004) was excluded as it did not report cohort-specific RR. Total number of studies in this meta-analysis: twenty-three for highest $v$. lowest MDS and twenty-five for per SD MDS increments. 


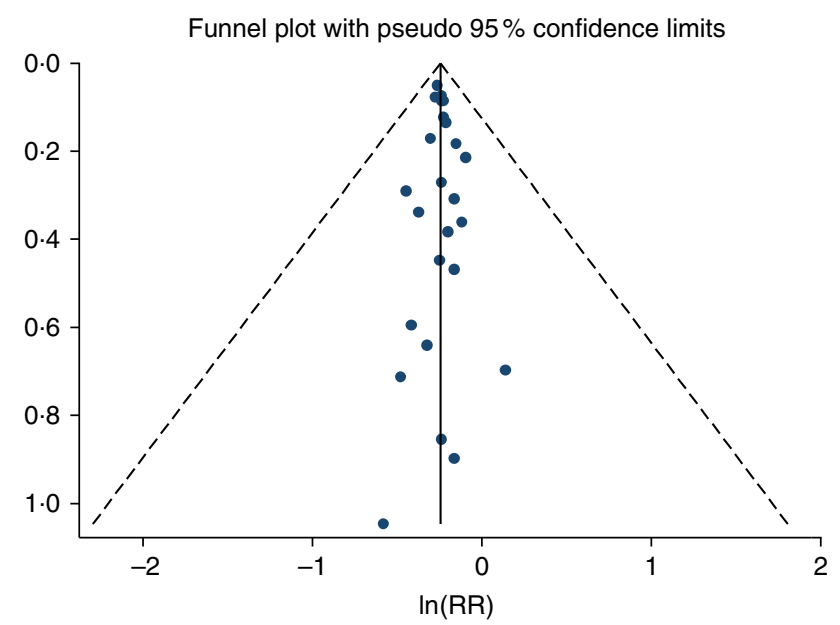

Fig. 4. Funnel plot for the assessment of publication bias in the studies included in the meta-analysis of mortality in association with the highest $v$. the lowest level of Mediterranean diet score ( $P$ for Egger test 0.67; $P$ for Begg's test 0.83). Dashed diagonal lines indicate $95 \% \mathrm{Cl}$.

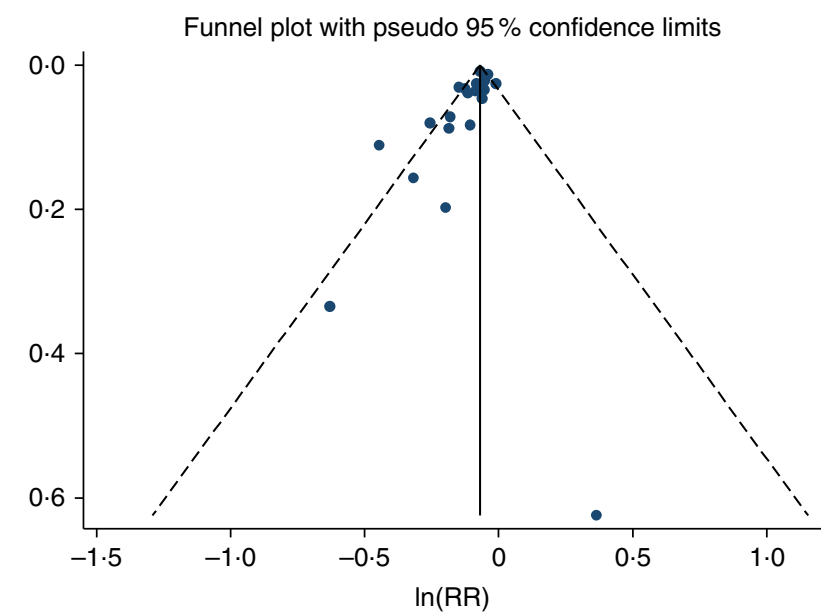

Fig. 5. Funnel plot for the assessment of publication bias in the studies included in the meta-analysis of mortality per one study-specific standard deviation increment in Mediterranean diet score $(P$ for Egger test $<0.01 ; P$ for Begg's test 0.01). Dashed diagonal lines indicate $95 \% \mathrm{Cl}$ ).

Table 3. Summary relative risks (RR) and $95 \% \mathrm{Cl}$ of all-cause mortality for the above $v$. below the study-specific median intake in each of the components of the Mediterranean diet (MedDiet)

\begin{tabular}{lccc}
\hline & \multicolumn{3}{c}{ Above $v$. below median intake of component } \\
\cline { 2 - 4 } MedDiet components & Number of studies & RR & $95 \% \mathrm{Cl}$ \\
\hline Lipid ratio & 6 & 0.98 & $0.93,1.04$ \\
Vegetables & 9 & 0.94 & $0.89,0.98$ \\
Legumes & 7 & 1.00 & $0.94,1.07$ \\
Fruit & 9 & 0.88 & $0.83,0.94$ \\
Cereals & 9 & 0.95 & $0.91,1.00$ \\
Fish & 9 & 1.01 & $0.96,1.06$ \\
Alcohol & 4 & 0.86 & $0.77,0.97^{\star}$ \\
& 6 & 0.95 & $0.83,1.08 \dagger$ \\
Dairy products & 9 & 1.05 & $0.98,1.12$ \\
Meat and products & 9 & 1.07 & $1.01,1.13$ \\
\hline
\end{tabular}

${ }^{*}$ Moderate $v$. none/excess consumption.

† Above $v$. below study specific median.
0.91 (95\% CI 0.89, 0.93) associated with a 2-point increment in MDS with moderate heterogeneity. Although previous studies have estimated RR for either continuous increments or categorical comparisons of MDS, we have chosen to provide RR separately for the two approaches, avoiding making any assumptions for transforming the reported RR (i.e. from expressing continuous increments to reflecting categorical comparisons of MDS and vice versa). Our approach adds to interpreting and communicating the results regarding the health benefits associated with the MedDiet: realistic increments of the adherence to the MedDiet result in a reduction of $8 \%$ in allcause mortality, whereas this survival benefit achieves its maximum ( $21 \%$ reduction in mortality rates) among adherers at the highest as compared with those in the lowest percentiles of MDS.

In our meta-analysis, we observed moderate heterogeneity across studies, similarly to previous meta-analyses ${ }^{(4)}$. When exploring this heterogeneity in strata of pre-defined covariates, none of the stratification variables had any appreciable influence on the summary RR, which were always indicative of inverse and significant associations of the MedDiet with mortality. Heterogeneity, however, was decreased, in a significant manner, among European as compared with US studies. Studies across USA and Europe may differ in definitions and components of MDS, intakes of foods included in MDS, correlations between components of MDS and so on. No appreciable heterogeneity across European studies was observed in our investigation, similarly to previous meta-analyses ${ }^{(58)}$, even though the reported associations were somewhat stronger in Mediterranean rather than in western and northern European countries. This is expected, as Mediterranean populations are likely to have greater adherence to MedDiet scores. The constructing algorithm underlying MedDiet indices, which usually results in different MDS ranges of scales, did not seem to influence the results of our meta-analysis. Studies published before 2011 were fewer and smaller with more imprecise RR as compared with the large body of studies published after 2011. Nevertheless, heterogeneity by year of publication (before/after 2011) was not statistically significant.

The beneficial effect of the MedDiet on several health outcomes has been often attributed to constituents such as vitamins, minerals, fibre and antioxidants abundant in foods of plant origin, ethanol and polyphenols in wine (the most frequent type of alcoholic beverage consumed in the traditional MedDiet) and antioxidative and anti-oncogenic effects of oleic acid abundant in olive oil, highly consumed in the MedDiet $^{(59-61)}$. In our study, we attempted to evaluate the contribution of each component on the association with mortality of the MedDiet in toto, by performing suitable meta-analyses. Examination of the summary mortality ratios revealed inverse or null associations for higher intakes of all MedDiet components except for meat and dairy intakes, for which the associations were positive, as reflected in the scores. Moreover, our results indicated highest protection for moderate ( $v$. excessive or minimal) alcohol consumption (14\%), followed by above ( $v$. below)-the-median consumption of fruit (12\%). Low (belowthe-median) consumption of meat and dairy products and high (above-the-median) consumption of vegetables and cereals 
(a)

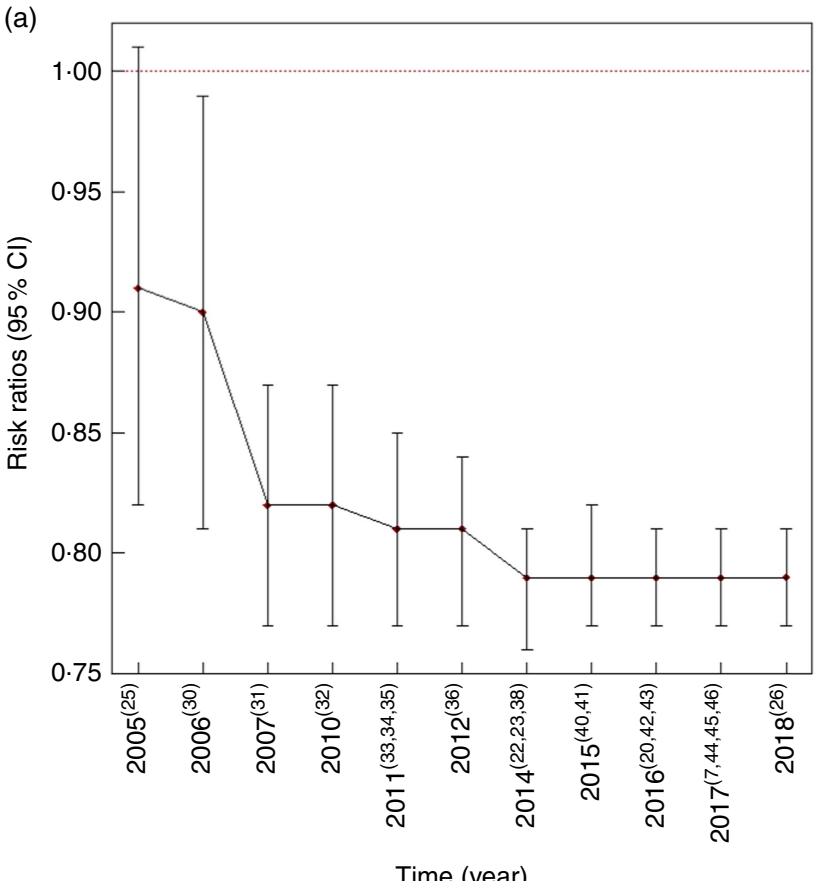

(b)

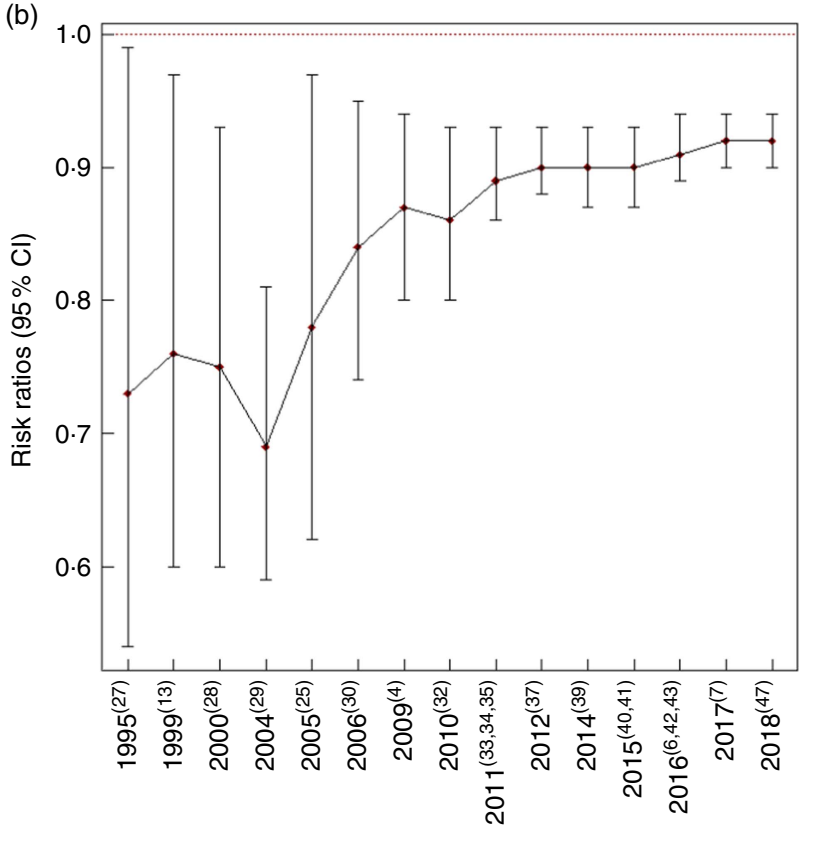

Time (year)

Fig. 6. (a) Cumulative meta-analysis of cohort studies on the Mediterranean Diet (MedDiet) (highest $v$. lowest category in each study) and mortality. (b) Cumulative meta-analysis of cohort studies on the MedDiet (per one study-specific standard deviation increment) and mortality. Diamonds are the estimated summary relative risks and error bars are the associated $95 \% \mathrm{Cl}$, by year of publication. References of studies included in the cumulative meta-analyses are shown in parentheses.

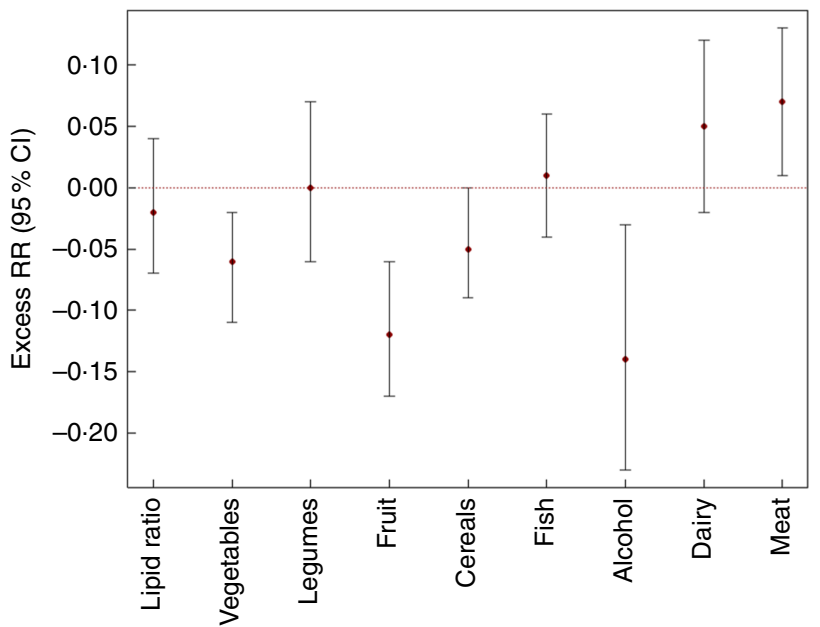

Fig. 7. Summary excess relative risk (RR) and $95 \% \mathrm{Cl}$ of mortality associated with each of the components of the Mediterranean diet being above $v$. below the study-specific median intake (lipid ratio, vegetables, fruit, legumes, cereals, dairy products, meat and products, fish) or for moderate $v$. none/excess consumption (alcohol). The combined hazard ratio and $95 \% \mathrm{Cl}$ were calculated using the random-effects models.

were associated with lower mortality rates of about 5-6\%. The association with mortality of the lipid ratio was minimal (2\%), whereas consumption of fish and seafood, as well as of legumes, was not associated with mortality. The importance of alcohol in the association of MDS with mortality may be partly owing to the more valid, as compared with the rest of the MedDiet components, reporting of habitual intake of alcoholic beverages. On the other hand, fruit and vegetables, as well as meat and meat products, are foods widely consumed in the countries included in this meta-analysis and therefore a 'true' association with mortality may be more easily detected as compared with other non-frequently consumed foods in the non-Mediterranean countries, such as legumes and olive oil (included in the lipid ratio).

A few studies have investigated the contribution of the MedDiet components in the association of this diet with mortality. An empirical investigation by Trichopoulou et al. ${ }^{(7)}$ (included in the current meta-analysis) based on the Greek EPIC also highlighted the high importance of moderate ( $v$. excessive or minimal) alcohol intake and the neutral contribution of fish intake to the association of the MedDiet with mortality. Although the magnitudes of the RR in that study were, naturally, different from our summary $R R$, the order of the relative importance of the different components was quite similar in their study and ours.

Our results regarding the contribution of the individual components to the overall association of the MedDiet with mortality should be interpreted with caution as (1) intakes of the indicated foods/nutrients have been collected with different methods and differ (on average) across studies included in the meta-analysis, (2) different number of studies are used in the meta-analysis of each of the individual components and (3) definitions of the foods comprising the MedDiet components are different across studies (e.g. only whole-grain cereals are used in the alternate MDS, but all types of cereals are included in the original MDS). Nevertheless, the summary RR for the nine MedDiet components are generally in agreement with the current literature. Several studies have reported the survival benefit 
of moderate alcohol intake ${ }^{(62)}$, of higher intakes of vegetables, fruit and nuts ${ }^{(63,64)}$ and of low/no intake of meat (especially red meat) and meat products ${ }^{(65,66)}$. For dairy products, a recent meta-analysis ${ }^{(5)}$ did not find an association with mortality. For cereals, results are controversial, as they include whole-grain cereals that have been found to be inversely associated with mortality $^{(5,67)}$, as well as refined grains for which no or a moderate direct association was found in previous metaanalyses $^{(5,68)}$. Fish and legume intakes have been inversely associated with mortality ${ }^{(5,69,70)}$. In our study, we did not detect an association with mortality for these two food groups, probably owing to their low intakes evident in the studies included in the component-specific meta-analyses. We did not find an appreciable influence in mortality of the lipid ratio intake, either. Although this ratio was proposed originally to reflect a high monounsaturated (mainly owing to high consumption of olive oil in the MedDiet) as opposed to saturated lipid intake, this is not always the case: in many studies undertaken in countries that culturally do not consume olive oil, monounsaturated lipid intake can be of animal origin, whereas polyunsaturated lipid intakes are usually added in the nominator in order to reflect lipid intake from plant origin. In a metaanalysis of Schwingshackl \& Hoffmann ${ }^{(71)}$ examining the role of MUFA (from plant and animal origin) and olive oil on mortality, the most consistent findings for reduced mortality were for olive oil intake.

Major strengths of our study are the thorough systematic review, allowing us to estimate the association of the MedDiet with all-cause mortality by comparing the extremes of adherence with this diet, as well as for smaller, more easily achievable changes in habitual intakes for those who wish to follow this diet. To our knowledge, this is the first study evaluating the relative merit of the individual components in the overall association of the MedDiet with mortality ${ }^{(5)}$. In all, two other meta-analyses have also looked at the individual components of the MedDiet in relation to incidence of, and mortality from, $\mathrm{CVD}^{(72)}$ and cancer ${ }^{(5)}$. It is difficult to compare the results of these investigations with ours owing to differences in the studies included, the end points used and the different contrasting categories used for each component. Nevertheless, the aforementioned investigations also found inverse associations for the incidence of/mortality from CVD and cancer with respect to higher intake of vegetables and fruit, and a positive association with respect to higher meat intake (albeit not statistically significant). Schwingshackl et al. ${ }^{(5)}$ also found moderate ethanol consumption being associated with a reduction in cancer incidence/mortality. On the other hand, Grosso et al. ${ }^{(72)}$ found a neutral effect of alcohol on cardiovascular incidence/mortality (0.97, $95 \%$ CI 0.88 1.07) but they have combined RR, which refer both to high $v$. low and moderate $v$. other consumptions of alcohol, and therefore their results are not directly comparable to ours.

However, some limitations should also be acknowledged. The studies included in our meta-analysis may have various sources of bias. Misclassification of consumption of the MedDiet components owing to self-reported assessment is likely in the original studies, but this would be non-differential among cohort studies where information is collected long time before mortality.
Another limitation is that adherence to the MedDiet has been evaluated only at recruitment, and individual diet may change over time especially in prospective studies with long follow-up. Nevertheless, a recent study, with a substantial sample size, that used a superior design with repeated measurements of diet and examined changes in adherence to the MedDiet during a very long follow-up also showed a benefit in mortality ${ }^{(15)}$.

Heterogeneity among RR associated with MDS was not negligible; however, the consistency of our findings with those reported in other meta-analyses of the MedDiet in relation to various health outcomes ${ }^{(6)}$ reassures the accuracy of our results. With respect to confounding, the well-accepted risk factors for mortality were controlled for in many studies, especially the most recent ones, and we used multivariate RR adjusted for all available covariates in our meta-analyses. Finally, the estimated $\mathrm{RR}$ referring to the individual components of the MedDiet were based on country-specific medians, which differ across countries, although the use of random-effects models may overcome this problem up to a certain point.

In conclusion, in our systematic meta-analyses of cohort studies, we confirmed the inverse association of the MedDiet with mortality among adults and we provided evidence regarding the contribution of the components of this diet in the overall association. Although the survival benefits of the MedDiet in toto are important from a holistic and public health point of view, understanding which components most influence the protective role of this diet seems also important in order to better promote the MedDiet as a healthy dietary pattern, as well as to propose changes in the intakes of the components of this diet that can most effectively lower mortality.

\section{Acknowledgements}

This research received no grant from any funding agency or from commercial or not-for-profit sectors.

C. B. was responsible for formulating the research questions and designing of the study. D. E., V. B. and C. B. carried out the literature search and critically reviewed the selected publications. D. E. performed the statistical analysis. D. E., V. B. and C. B. were responsible for writing the paper. D. E., V. B., A. T., C. L. V. and C. B. critically reviewed the manuscript. All authors have read and approved the final manuscript.

The authors declare that there are no conflicts of interest.

\section{References}

1. Martínez-González MA \& Bes-Rastrollo M (2014) Dietary patterns, Mediterranean diet, and cardiovascular disease. Curr Opin Lipidol 25, 20-26.

2. Schwingshackl L \& Hoffmann G (2014) Mediterranean dietary pattern, inflammation and endothelial function: a systematic review and meta-analysis of intervention trials. Nutr Metab Cardiovasc Dis 24, 929-939.

3. Gay HC, Rao SG, Vaccarino V, et al. (2016) Effects of different dietary interventions on blood pressure: systematic review and meta-analysis of randomized controlled trials. Hypertension 67, 733-739.

4. Sofi F, Macchi C, Abbate R, et al. (2014) Mediterranean diet and health status: an updated meta-analysis and a proposal for 
a literature-based adherence score. Public Health Nutr 17, 2769-2782.

5. Schwingshackl L, Schwedhelm C, Galbete C, et al. (2017) Adherence to Mediterranean diet and risk of cancer: an updated systematic review and meta-analysis. Nutrients 9 , E1063.

6. Dinu M, Pagliai G, Casini A, et al. (2018) Mediterranean diet and multiple health outcomes: an umbrella review of metaanalyses of observational studies and randomised trials. Eur J Clin Nutr 72, 30-43.

7. Trichopoulou A, Bamia C \& Trichopoulos D (2009) Anatomy of health effects of Mediterranean diet: Greek EPIC prospective cohort study. BMJ 338, b2337.

8. Martínez-González MA, Guillén-Grima F, De Irala $\mathrm{J}$, et al. (2012) The Mediterranean diet is associated with a reduction in premature mortality among middle-aged adults. J Nutr $\mathbf{1 4 2}$, 1672-1678.

9. Tong TY, Wareham NJ, Khaw KT, et al. (2016) Prospective association of the Mediterranean diet with cardiovascular disease incidence and mortality and its population impact in a non-Mediterranean population: the EPIC-Norfolk study. BMC Med 14, 135.

10. Stefler D, Malyutina S, Kubinova R, et al. (2017) Mediterranean diet score and total and cardiovascular mortality in Eastern Europe: the HAPIEE study. Eur J Nutr 56, 421-429.

11. Bloomfield HE, Koeller E, Greer N, et al. (2016) Effects on health outcomes of a Mediterranean diet with no restriction on fat intake: a systematic review and meta-analysis. Ann Intern Med 165, 491-500.

12. Zazpe I, Sanchez-Tainta A, Toledo E, et al. (2014) Dietary patterns and total mortality in a Mediterranean cohort: the SUN project. I Acad Nutr Diet 114, 37-47.

13. Martínez-González MA, Zazpe I, Razquin C, et al. (2015) Empirically-derived food patterns and the risk of total mortality and cardiovascular events in the PREDIMED study. Clin Nutr 34, 859-867.

14. Menotti A, Puddu PE, Maiani G, et al. (2016) Cardiovascular and other causes of death as a function of lifestyle habits in a quasi extinct middle-aged male population. A 50-year follow-up study. Int J Cardiol 210, 173-178.

15. Kouris-Blazos A \& Itsiopoulos C (2014) Low all-cause mortality despite high cardiovascular risk in elderly Greek-born Australians: attenuating potential of diet? Asia Pac J Clin Nutr 23, 532-544.

16. Kouris-Blazos A, Gnardellis C, Wahlqvist ML, et al. (1999) Are the advantages of the Mediterranean diet transferable to other populations? A cohort study in Melbourne, Australia. Br J Nutr 82, 57-61.

17. Sotos-Prieto M, Bhupathiraju SN, Mattei J, et al. (2017) Association of changes in diet quality with total and cause-specific mortality. N Engl J Med 377, 143-153.

18. Panagiotakos DB, Georgousopoulou EN, Pitsavos C, et al. (2015) Ten-year (2002-2012) cardiovascular disease incidence and all-cause mortality, in urban Greek population: the ATTICA Study. Int J Cardiol 180, 178-184.

19. Guasch-Ferré M, Salas-Salvadó J, Ros E, et al. (2017) The PREDIMED trial, Mediterranean diet and health outcomes: how strong is the evidence? Nutr Metab Cardiovasc Dis 27, 624-632.

20. Ebell MH \& Grad R (2017) Top 20 research studies of 2016 for primary care physicians. Am Fam Physician 95, 572-579.

21. Rautiainen S, Gaziano JM, Christen WG, et al. (2017) Effect of baseline nutritional status on long-term multivitamin use and cardiovascular disease risk: a secondary analysis of the Physicians' Health Study II randomized clinical trial. JAMA Cardiol 2, 617-625.
22. Harmon BE, Boushey CJ, Shvetsov YB, et al. (2015) Associations of key diet-quality indexes with mortality in the multiethnic cohort: the Dietary Patterns Methods Project. Am J Clin Nutr 101, 587-597.

23. Shvetsov YB, Harmon BE, Ettienne R, et al. (2016) The influence of energy standardisation on the alternate Mediterranean diet score and its association with mortality in the multiethnic cohort. Br J Nutr 116, 1592-1601.

24. Liese AD, Krebs-Smith SM, Subar AF, et al. (2015) The Dietary Patterns Methods Project: synthesis of findings across cohorts and relevance to dietary guidance. J Nutr $\mathbf{1 4 5}$, 393-402.

25. George SM, Ballard-Barbash R, Manson JE, et al. (2014) Comparing indices of diet quality with chronic disease mortality risk in postmenopausal women in the Women's Health Initiative Observational Study: evidence to inform national dietary guidance. Am J Epidemiol 180, 616-625.

26. Reedy J, Krebs-Smith SM, Miller PE, et al. (2014) Higher diet quality is associated with decreased risk of all-cause, cardiovascular disease, and cancer mortality among older adults. J Nutr 144, 881-889.

27. Lassale C, Gunter MJ, Romaguera D, et al. (2016) Diet quality scores and prediction of all-cause, cardiovascular and cancer mortality in a pan-European cohort study. PLOS ONE 11, e0159025.

28. Trichopoulou A, Orfanos P, Norat T, et al. (2005) Modified Mediterranean diet and survival: EPIC-elderly prospective cohort study. BMJ 330, 991.

29. Alvarez-Alvarez I, Zazpe I, Pérez de Rojas J, et al. (2018) Mediterranean diet, physical activity and their combined effect on all-cause mortality: the Seguimiento Universidad de Navarra (SUN) cohort. Prev Med 106, 45-52.

30. Trichopoulou A, Kouris-Blazos A, Wahlqvist ML, et al. (1995) Diet and overall premature mortality in men and women survival in elderly people. BMJ 311, 1457-1460.

31. Lasheras C, Fernandez S \& Patterson AM (2000) Mediterranean diet and age with respect to overall survival in institutionalized, nonsmoking elderly people. Am J Clin Nutr $\mathbf{7 1}$, 987-992.

32. Knoops KT, de Groot LC, Kromhout D, et al. (2004) Mediterranean diet, lifestyle factors, and 10-year mortality in elderly European men and women: the HALE project. JAMA 292, $1433-1439$

33. Lagiou P, Trichopoulos D, Sandin S, et al. (2006) Mediterranean dietary pattern and mortality among young women: a cohort study in Sweden. Br J Nutr 96, 384-392.

34. Mitrou PN, Kipnis V, Thiébaut AC, et al. (2007) Mediterranean dietary pattern and prediction of all-cause mortality in a US population: results from the NIH-AARP Diet and Health Study. Arch Intern Med 167, 2461-2468.

35. Sjögren P, Becker W, Warensjö E, et al. (2010) Mediterranean and carbohydrate-restricted diets and mortality among elderly men: a cohort study in Sweden. Am J Clin Nutr 92, 967-974.

36. Tognon G, Rothenberg E, Eiben G, et al. (2011) Does the Mediterranean diet predict longevity in the elderly? A Swedish perspective. Age (Dordr) 33, 439-450.

37. Buckland G, Agudo A, Travier N, et al. (2011) Adherence to the Mediterranean diet reduces mortality in the Spanish cohort of the European Prospective Investigation into Cancer and Nutrition (EPIC-Spain). Br J Nutr 106, 1581-1591.

38. van den Brandt PA (2011) The impact of a Mediterranean diet and healthy lifestyle on premature mortality in men and women. Am J Clin Nutr 94, 913-920.

39. McNaughton SA, Bates CJ \& Mishra GD (2012) Diet quality is associated with all-cause mortality in adults aged 65 years and older. J Nutr 142, 320-325. 
40. Tognon G, Nilsson LM, Lissner L, et al. (2012) The Mediterranean diet score and mortality are inversely associated in adults living in the subarctic region. J Nutr 142, 1547-1553.

41. Cuenca-García M, Artero EG, Sui X, et al. (2014) Dietary indices, cardiovascular risk factors and mortality in middleaged adults: findings from the Aerobics Center Longitudinal Study. Ann Epidemiol 24, 297-303.e2.

42. Tognon G, Lissner L, Sæbye D, et al. (2014) The Mediterranean diet in relation to mortality and CVD: a Danish cohort study. Br J Nutr 111, 151-159.

43. Vormund K, Braun J, Rohrmann S, et al. (2015) Mediterranean diet and mortality in Switzerland: an alpine paradox? Eur J Nutr 54, 139-148.

44. Prinelli F, Yannakoulia M, Anastasiou CA, et al. (2015) Mediterranean diet and other lifestyle factors in relation to 20-year all-cause mortality: a cohort study in an Italian population. $\mathrm{BrJ}$ Nutr 113, 1003-1011.

45. Bellavia A, Tektonidis TG, Orsini N, et al. (2016) Quantifying the benefits of Mediterranean diet in terms of survival. Eur J Epidemiol 31, 527-530.

46. Bo S, Ponzo V, Goitre I, et al. (2016) Predictive role of the Mediterranean diet on mortality in individuals at low cardiovascular risk: a 12-year follow-up population-based cohort study. J Transl Med 14, 91.

47. Limongi F, Noale M, Gesmundo A, et al. (2017) Adherence to the Mediterranean diet and all-cause mortality risk in an elderly Italian population: data from the ILSA study. $J$ Nutr Health Aging 21, 505-513.

48. Whalen KA, Judd S, McCullough ML, et al. (2017) Paleolithic and Mediterranean diet pattern scores are inversely associated with all-cause and cause-specific mortality in adults. $J$ Nutr 147, 612-620.

49. Franzon K, Byberg L, Sjögren P, et al. (2017) Predictors of independent aging and survival: a 16-year follow-up report in octogenarian men. J Am Geriatr Soc 65, 1953-1960.

50. Shah NS, Leonard D, Finley CE, et al. (2018) Dietary patterns and long-term survival: a retrospective study of healthy primary care patients. Am J Med 131, 48-55.

51. Trichopoulou A, Costacou T, Bamia C, et al. (2003) Adherence to a Mediterranean diet and survival in a Greek population. $N$ Engl J Med 348, 2599-2608.

52. Georgousopoulou EN, Kastorini CM, Milionis HJ, et al. (2014) Association between Mediterranean diet and non-fatal cardiovascular events, in the context of anxiety and depression disorders: a case/case-control study. Hellenic J Cardiol 55, 24-31

53. DerSimonian R \& Laird N (1986) Meta-analysis in clinical trials. Control Clin Trials 7, 177-188.

54. Greenland S (1987) Quantitative methods in the review of epidemiologic literature. Epidemiol Rev 9, 1-30.

55. Higgins JP \& Thompson SG (2002) Quantifying heterogeneity in a meta-analysis. Stat Med 21, 1539-1558.

56. Stuck AE, Rubenstein LZ \& Wieland D (1998) Bias in metaanalysis detected by a simple, graphical test. Asymmetry detected in funnel plot was probably due to true heterogeneity. BMJ 316, 469, author reply 470-471.

57. Macaskill P, Walter SD \& Irwig L (2001) A comparison of methods to detect publication bias in meta-analysis. Stat Med 20, 641-654.
58. Sofi F, Abbate R, Gensini GF, et al. (2010) Accruing evidence on benefits of adherence to the Mediterranean diet on health: an updated systematic review and meta-analysis. Am J Clin Nutr 92, 1189-1196.

59. Menendez JA \& Lupu R (2006) Mediterranean dietary traditions for the molecular treatment of human cancer: antioncogenic actions of the main olive oil's monounsaturated fatty acid oleic acid (18:1n-9). Curr Pharm Biotechnol 7, 495-502.

60. Dedoussis GV, Kanoni S, Mariani E, et al. (2008) Mediterranean diet and plasma concentration of inflammatory markers in old and very old subjects in the ZINCAGE population study. Clin Chem Lab Med 46, 990-996.

61. Pelucchi C, Bosetti C, Negri E, et al. (2011) Olive oil and cancer risk: an update of epidemiological findings through 2010. Curr Pharm Des 17, 805-812.

62. Di Castelnuovo A, Costanzo S, Bagnardi V, et al. (2006) Alcohol dosing and total mortality in men and women: an updated meta-analysis of 34 prospective studies. Arch Intern Med 166, 2437-2445.

63. Wang X, Ouyang Y, Liu J, et al. (2014) Fruit and vegetable consumption and mortality from all causes, cardiovascular disease, and cancer: systematic review and dose-response meta-analysis of prospective cohort studies. BMJ 349, g4490.

64. Grosso G, Yang J, Marventano S, et al. (2015) Nut consumption on all-cause, cardiovascular, and cancer mortality risk: a systematic review and meta-analysis of epidemiologic studies. Am J Clin Nutr 101, 783-793.

65. Abete I, Romaguera D, Vieira AR, et al. (2014) Association between total, processed, red and white meat consumption and all-cause, CVD and IHD mortality: a meta-analysis of cohort studies. Br J Nutr 112, 762-775.

66. Wang X, Lin X, Ouyang YY, et al. (2016) Red and processed meat consumption and mortality: dose-response metaanalysis of prospective cohort studies. Public Health Nutr 19, 893-905.

67. Aune D, Keum N, Giovannucci E, et al. (2016) Whole grain consumption and risk of cardiovascular disease, cancer, and all cause and cause specific mortality: systematic review and dose-response meta-analysis of prospective studies. BMJ $\mathbf{3 5 3}$, i2716.

68. Hajishafiee M, Saneei P, Benisi-Kohansal S, et al. (2016) Cereal fibre intake and risk of mortality from all causes, CVD, cancer and inflammatory diseases: a systematic review and metaanalysis of prospective cohort studies. Br J Nutr 116, 343-352.

69. Zhao LG, Sun JW, Yang Y, et al. (2016) Fish consumption and all-cause mortality: a meta-analysis of cohort studies. Eur J Clin Nutr 70, 155-161.

70. Marventano S, Izquierdo Pulido M, Sánchez-González C, et al. (2017) Legume consumption and CVD risk: a systematic review and meta-analysis. Public Health Nutr 20, 245-254.

71. Schwingshackl L \& Hoffmann G (2014) Monounsaturated fatty acids, olive oil and health status: a systematic review and meta-analysis of cohort studies. Lipids Health Dis 13, 154 .

72. Grosso G, Marventano S, Yang J, et al. (2017) A comprehensive meta-analysis on evidence of mediterranean diet and cardiovascular disease: are individual components equal? Crit Rev Food Sci Nutr 57, 3218-3232. 